\title{
Determination of Permeability Tensors for Two-Phase Flow in Homogeneous Porous Media: Theory
}

\author{
DIDIER LASSEUX and MICHEL QUINTARD
}

L.E.P.T.-ENSAM (UA CNRS), Esplanade des Arts et Métiers, 33405 Talence cedex, France. email: lasseux@lept-ensam.u-bordeaux.fr

STEPHEN WHITAKER

Department of Chemical Engineering and Material Science, University of California at Davis, Davis, CA 95616, U.S.A.

\begin{abstract}
In this paper we continue previous studies of the closure problem for two-phase flow in homogeneous porous media, and we show how the closure problem can be transformed to a pair of Stokes-like boundary-value problems in terms of 'pressures' that have units of length and 'velocities' that have units of length squared. These are essentially geometrical boundary value problems that are used to calculate the four permeability tensors that appear in the volume averaged Stokes' equations. To determine the geometry associated with the closure problem, one needs to solve the physical problem; however, the closure problem can be solved using the same algorithm used to solve the physical problem, thus the entire procedure can be accomplished with a single numerical code.
\end{abstract}

Key words: homogeneous porous media, two-phase flow, volume averaging, permeability tensors

\section{Nomenclature}

$\mathbf{a}_{\alpha \kappa} \quad$ a vector that maps $\boldsymbol{\mu}_{\alpha}\left\langle\mathbf{v}_{\kappa}\right\rangle^{\kappa}$ onto $\tilde{p}_{\alpha}, \mathrm{m}^{-1}$.

A $\quad$ a tensor that maps $\left\langle\mathbf{v}_{\kappa}\right\rangle^{\kappa}$ onto $\tilde{\mathbf{v}}_{\alpha}$.

$\mathcal{A}_{\alpha \kappa} \quad$ area of the $\alpha-\kappa$ interface contained within the macroscopic region, $\mathrm{m}^{2}$.

$\mathcal{A}_{\alpha e} \quad$ area of the $\alpha$-phase entrances and exits contained within the macroscopic region, $\mathrm{m}^{2}$.

$A_{\alpha \kappa} \quad$ area of the $\alpha-\kappa$ interface contained within the averaging volume, $\mathrm{m}^{2}$.

$A_{\alpha e} \quad$ area of the $\alpha$-phase entrances and exits contained within the averaging volume, $\mathrm{m}^{2}$.

Bo Bond number $\left(=\left(\rho_{\beta}-\rho_{\gamma}\right) g \ell^{2} / \sigma\right)$.

$\mathrm{Ca}$ capillary number $(=\mu\langle v\rangle / \sigma)$.

g gravitational acceleration, $\mathrm{m} / \mathrm{s}^{2}$.

$H$ mean curvature, $\mathrm{m}^{-1}$.

I unit tensor.

$\mathbf{K}_{\alpha} \quad$ permeability tensor for the $\alpha$-phase, $\mathrm{m}^{2}$.

$\mathbf{K}_{\alpha \kappa} \quad$ viscous drag tensor that maps $\left\langle\mathbf{v}_{\kappa}\right\rangle$ onto $\left\langle\mathbf{v}_{\alpha}\right\rangle$.

$\mathbf{K}_{\alpha \alpha}^{*} \quad$ dominant permeability tensor that maps $\left(\frac{\nabla\left\langle p_{\alpha}\right\rangle^{\alpha}-\rho_{\alpha} \mathbf{g}}{\mu_{\alpha}}\right)$ onto $\left\langle\mathbf{v}_{\alpha}\right\rangle, \mathrm{m}^{2}$.

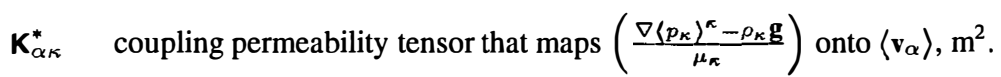


$\ell_{\alpha} \quad$ characteristic length scale for the $\alpha$-phase, $\mathrm{m}$.

$\ell \quad$ characteristic length scale representing both $\ell_{\beta}$ and $\ell_{\gamma}, \mathrm{m}$.

$L \quad$ characteristic length scale for volume averaged quantities, $\mathrm{m}$.

$\mathbf{n}_{\alpha \kappa} \quad$ unit normal vector directed from the $\alpha$-phase toward the $\kappa$-phase.

$\mathbf{n}_{\beta} \quad$ unit normal vector representing both $\mathbf{n}_{\beta \sigma}$ and $\mathbf{n}_{\beta \gamma}$.

$\mathbf{n}_{\gamma} \quad$ unit normal vector representing both $\mathbf{n}_{\gamma \sigma}$ and $\mathbf{n}_{\gamma \beta}$.

$P_{\alpha} \quad$ pressure in the $\alpha$-phase, $\mathrm{N} / \mathrm{m}^{2}$.

$\left\langle p_{\alpha}\right\rangle \quad$ superficial average pressure in the $\alpha$-phase, $\mathrm{N} / \mathrm{m}^{2}$.

$\left\langle p_{\alpha}\right\rangle^{\alpha}$ intrinsic average pressure in the $\alpha$-phase, $\mathrm{N} / \mathrm{m}^{2}$.

$\tilde{p}_{\alpha} \quad p_{\alpha}-\left\langle p_{\alpha}\right\rangle^{\alpha}$, spatial deviation pressure for the $\alpha$-phase, $\mathrm{N} / \mathrm{m}^{2}$.

$r_{0} \quad$ radius of the averaging volume, $\mathrm{m}$.

r position vector, $\mathrm{m}$.

$t$ time, $s$.

$\mathbf{v}_{\alpha} \quad$ fluid velocity in the $\alpha$-phase, $\mathrm{m} / \mathrm{s}$.

$\left\langle\mathrm{v}_{\alpha}\right\rangle \quad$ superficial average velocity in the $\alpha$-phase, $\mathrm{m} / \mathrm{s}$.

$\left\langle\mathbf{v}_{\alpha}\right\rangle^{\alpha}$ intrinsic average velocity in the $\alpha$-phase, $\mathrm{m} / \mathrm{s}$.

$\tilde{\mathbf{v}}_{\alpha} \quad \mathbf{v}_{\alpha}-\left\langle\mathbf{v}_{\alpha}\right\rangle^{\alpha}$, spatial deviation velocity in the $\alpha$-phase, $\mathrm{m} / \mathrm{s}$.

$V_{\alpha} \quad$ volume of the $\alpha$-phase contained within the averaging volmue, $\mathrm{m}^{3}$.

$\mathcal{V} \quad$ averaging volume, $\mathrm{m}^{3}$.

\section{Greek Symbols}

$\varepsilon_{\alpha} \quad V_{\alpha} / \mathcal{V}$, volume fraction of the $\alpha$-phase.

$\mu_{\alpha} \quad$ viscosity of the $\alpha$-phase, $\mathrm{Ns} / \mathrm{m}^{2}$.

$\rho_{\alpha} \quad$ density of the $\alpha$-phase, $\mathrm{kg} / \mathrm{m}^{3}$.

$\sigma \quad$ surface tension, $\mathrm{N} / \mathrm{m}$.

$\tau_{\alpha} \quad \mu\left(\nabla \mathbf{v}_{\alpha}+\nabla \mathbf{v}_{\alpha}^{T}\right)$, viscous stress tensor for the $\alpha$-phase, $\mathrm{N} / \mathrm{m}^{2}$.

\section{Introduction}

In previous studies of two-phase flow in homogeneous porous media, the volume averaged Stokes' equations were shown to have the form

$$
\begin{aligned}
& \left\langle\mathbf{v}_{\beta}\right\rangle=-\frac{\mathbf{K}_{\beta}}{\mu_{\beta}} \cdot\left(\nabla\left\langle p_{\beta}\right\rangle^{\beta}-\rho_{\beta} \mathbf{g}\right)+\mathbf{K}_{\beta \gamma} \cdot\left\langle\mathbf{v}_{\gamma}\right\rangle, \\
& \left\langle\mathbf{v}_{\gamma}\right\rangle=-\frac{\mathbf{K}_{\gamma}}{\mu_{\gamma}} \cdot\left(\nabla\left\langle p_{\gamma}\right\rangle^{\gamma}-\rho_{\gamma} \mathbf{g}\right)+\mathbf{K}_{\gamma \beta} \cdot\left\langle\mathbf{v}_{\beta}\right\rangle,
\end{aligned}
$$

in which $\mathbf{K}_{\beta}$ and $\mathbf{K}_{\gamma}$ are permeability tensors and $\mathbf{K}_{\beta \gamma}$ and $\mathbf{K}_{\gamma \beta}$ are viscous drag tensors. These forms of the volume averaged momentum equations were first postulated by Raats and Klute (1968) and later developed by Baveye and Sposito (1984). Closure problems have been derived (Whitaker, 1986, 1994) that can be used to predict the permeability tensors and to assess the importance of the viscous drag tensors. A qualitative examination of the latter suggests that they satisfy the 
condition $\mathbf{K}_{\beta \gamma} \cdot \mathbf{K}_{\gamma \beta}=\mathbf{O}(\mathbf{I})$; however, we have now developed an exact relation between these two tensors that is given by

$$
\mu_{\beta} \mathbf{K}_{\beta \gamma} \cdot \mathbf{K}_{\gamma}=\mu_{\gamma} \mathbf{K}_{\beta} \cdot \mathbf{K}_{\gamma \beta}^{T}
$$

and the details are presented in the appendix.

Equations (1.1) and (1.2) can be rearranged to obtain

$$
\begin{aligned}
& \left\langle\mathbf{v}_{\beta}\right\rangle=-\frac{\mathbf{K}_{\beta \beta}^{*}}{\mu_{\beta}} \cdot\left(\nabla\left\langle p_{\beta}\right\rangle^{\beta}-\rho_{\beta} \mathbf{g}\right)-\frac{\mathbf{K}_{\beta \gamma}^{*}}{\mu_{\gamma}} \cdot\left(\nabla\left\langle p_{\gamma}\right\rangle^{\gamma}-\rho_{\gamma} \mathbf{g}\right), \\
& \left\langle\mathbf{v}_{\gamma}\right\rangle=-\frac{\mathbf{K}_{\gamma \gamma}^{*}}{\mu_{\gamma}} \cdot\left(\nabla\left\langle p_{\gamma}\right\rangle^{\gamma}-\rho_{\gamma} \mathbf{g}\right)-\frac{\mathbf{K}_{\gamma \beta}^{*}}{\mu_{\beta}} \cdot\left(\nabla\left\langle p_{\beta}\right\rangle^{\beta}-\rho_{\beta} \mathbf{g}\right),
\end{aligned}
$$

in which $\mathbf{K}_{\beta \beta}^{*}, \mathbf{K}_{\beta \gamma}^{*}, \mathbf{K}_{\gamma \beta}^{*}$ and $\mathbf{K}_{\gamma \gamma}^{*}$ are referred to as permeability tensors. These tensors are determined by a transformed version of the closure problem that provides $\mathbf{K}_{\beta}, \mathbf{K}_{\gamma}, \mathbf{K}_{\beta \gamma}$ and $\mathbf{K}_{\gamma \beta}$, and it is this transformed closure problem that has led us to the result given by Equation (1.3). The form represented by Equations (1.4) and (1.5) is contained in the macroscopic momentum equations derived by Marle (1982) using the method of volume averaging, and by de Gennes (1983) who developed these equations starting from the concepts of irreversible thermodynamics. Equations (1.4) and (1.5) were also derived by Auriault (1987) using the method of spatial homogenization (Bensoussan et al., 1978), and an extensive discussion of the coupling effects has been given by Kalaydjian $(1988,1990)$.

The problem under consideration is illustrated in Figure 1 where we have shown a macroscopic region and an averaging volume in which the $\beta$ and $\gamma$-phases represent the two fluid phases and the $\sigma$-phase represents the rigid, solid phase. The generic macroscopic length-scale is indicated by $L$ and the characteristic lengthscales for the two fluid phases are represented by $\ell_{\beta}$ and $\ell_{\gamma}$. To be clear about what we mean by a homogeneous porous medium, we refer to the definition given by Quintard and Whitaker (1987) in a study of single-phase flow in heterogeneous porous media.

A porous medium is homogenous with respect to a given process and a given averaging volume when the effective transport coefficients in the volume averaged transport equations are independent of position. If the porous medium is not homogenous, it is heterogeneous.

Most practical problems of two-phase flow in porous media are associated with porous media that are heterogeneous with respect to the Darcy scale, thus any study of homogeneous porous media can provide only the starting point for a wide range of practical problems. By Darcy scale we mean the scale at which the no-slip boundary condition is joined to the Stokes' equations by volume averaging. It is uncertain at this point in time that the viscous drag tensors, $\mathbf{K}_{\beta \gamma}$ and $\mathbf{K}_{\gamma \beta}$, or the coupling permeability tensors, $\mathbf{K}_{\beta \gamma}^{*}$ and $\mathbf{K}_{\gamma \beta}^{*}$, play an important role in the description of two-phase flow in heterogeneous porous media. However, it is clear that 


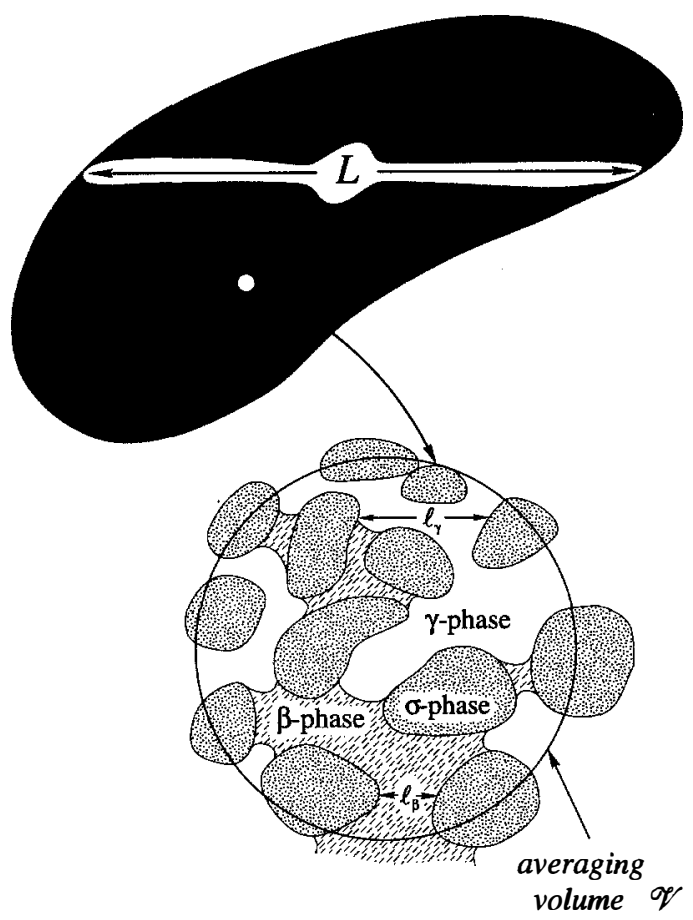

Figure 1. Macroscopic region and averaging volume.

these terms are important for certain two-phase flows such as flows in capillaries (Kalaydjian, 1990; Bacri et al., 1990), and flows in capillary networks (Rothman, 1990; Goode and Ramakrishnan, 1993). Other pore-scale numerical simulations confirm the importance of the exchange of momentum through the $\beta-\gamma$ interface (Danis and Jacquin, 1983; Danis and Quintard, 1984). Experimental measurement of these coefficients for flows in capillaries confirm theoretical predictions (Kalaydjian 1988, 1990; Kalaydjian and Legait 1987(a), 1987(b), 1988); however, the results of investigations for more complex media are not clear. Indirect measurements have been proposed and/or performed by Rose $(1988,1989)$, Bourbiaux and Kalaydjian (1990), Kalaydjian (1990), Mannseth (1991), and Bentsen and Manai (1993), and the results suggest that the coupling terms might be important for natural media. However, these measurements involve two different experiments for the same set of multiphase permeabilities, for instance, co-current and counter-current flows. Under these circumstances, the permeability tensors, at the same saturation, may differ because of the difference in the interface geometry and this makes it difficult to determine whether the coupling terms are important. On the other hand, recent direct measurements (Zarcone and Lenormand, 1994; Zarcone, 1994) suggest that the coupling terms are negligible for some classical natural media such as sand. Clearly the role of the coupling terms is still a matter of controversy and more theoretical and experimental analysis is required. In this work, we are concerned 
with the theoretical analysis, and we explore the closure problem that provides a direct method of calculating both the permeability tensors and the coupling viscous drag tensors.

The details of the physical process under consideration are described by the following boundary-value problem

$$
\begin{aligned}
& \nabla \cdot \mathbf{v}_{\beta}=0, \quad \text { in the } \beta \text {-phase, } \\
& 0=-\nabla p_{\beta}+\rho_{\beta} \mathbf{g}+\mu_{\beta} \nabla^{2} \mathbf{v}_{\beta}, \quad \text { in the } \beta \text {-phase, }
\end{aligned}
$$

B.C.1 $\mathbf{v}_{\beta}=0$, at $\mathcal{A}_{\beta \sigma}$,

B.C. $2 \quad \mathbf{v}_{\beta}=\mathbf{v}_{\gamma}, \quad$ at $\mathcal{A}_{\beta \gamma}$,

B.C.3 $-\mathbf{n}_{\beta \gamma} p_{\beta}+\mathbf{n}_{\beta \gamma} \cdot \tau_{\beta}$

$$
=-\mathbf{n}_{\beta \gamma} p_{\gamma}+\mathbf{n}_{\beta \gamma} \cdot \tau_{\gamma}+2 \sigma H \mathbf{n}_{\beta \gamma}, \quad \text { at } \mathcal{A}_{\beta \sigma},
$$

B.C. $4 \quad \mathbf{v}_{\gamma}=0, \quad$ at $\mathcal{A}_{\gamma \sigma}$,

$$
0=-\nabla p_{\gamma}+\rho_{\gamma} \mathbf{g}+\mu_{\gamma} \nabla^{2} \mathbf{v}_{\gamma}, \quad \text { in the } \gamma \text {-phase, }
$$

$$
\nabla \cdot \mathbf{v}_{\gamma}=0, \quad \text { in the } \gamma \text {-phase, }
$$

B.C.5 $\mathbf{v}_{\beta}=\mathbf{f}(t)$ at $\mathcal{A}_{\beta e}$,

B.C.6 $\mathbf{v}_{\gamma}=\mathbf{g}(t), \quad$ at $\mathcal{A}_{\gamma e}$.

Here we have used $\mathcal{A}_{\beta \gamma}$ to represent the $\beta$ - $\gamma$ interface contained within the macroscopic region illustrated in Figure 1, while $\mathcal{A}_{\beta e}$ and $\mathcal{A}_{\gamma e}$ represent the $\beta$ and $\gamma$-phase entrances and exits for that region. Even though most two-phase flows are unsteady, good arguments can be put forth in favor of the quasi-steady form of the Stokes' equations, and one can easily justify the incompressible form of the continuity equations.

To develop the volume averaged form of the continuity and momentum equations, we will make use of both superficial and intrinsic averages. For some quantity $\psi_{\beta}$ associated with the $\beta$-phase, the superficial average is defined by

$$
\left\langle\psi_{\beta}\right\rangle=\frac{1}{\mathcal{V}} \int_{V_{\beta}} \psi_{\beta} \mathrm{d} V
$$

Here $\mathcal{V}$ represents the averaging volume illustrated in Figure 1 and $V_{\beta}$ is the volume of the $\beta$-phase contained in the averaging volume. In addition to the superficial average, we will also make use of the intrinsic average that is given by

$$
\left\langle\psi_{\beta}\right\rangle^{\beta}=\frac{1}{V_{\beta}} \int_{V_{\beta}} \psi_{\beta} \mathrm{d} V
$$


These two average are obviously related according to

$$
\left\langle\psi_{\beta}\right\rangle=\varepsilon_{\beta}\left\langle\psi_{\beta}\right\rangle^{\beta}
$$

in which $\varepsilon_{\beta}$ is the volume fraction of the $\beta$-phase given explicitly as

$$
\varepsilon_{\beta}=\frac{V_{\beta}(t)}{\mathcal{V}}
$$

In addition to well-defined averages, we will need to make use of the averaging theorem (Howes and Whitaker, 1985) for a three-phase system. This can be expressed as

$$
\left\langle\nabla \psi_{\beta}\right\rangle=\nabla\left\langle\psi_{\beta}\right\rangle+\frac{1}{\mathcal{V}} \int_{A_{\beta \sigma}} \mathbf{n}_{\beta \sigma} \psi_{\beta} \mathrm{d} A+\frac{1}{\mathcal{V}} \int_{A_{\beta \gamma}} \mathbf{n}_{\beta \gamma} \psi_{\beta} \mathrm{d} A
$$

\subsection{CONTINUITY EQUATION}

We begin with the continuity equation for the $\beta$-phase and express the superficial volume average as

$$
\frac{1}{\mathcal{V}} \int_{V_{\beta}(t)} \nabla \cdot \mathbf{v}_{\beta} \mathrm{d} V=\left\langle\nabla \cdot \mathbf{v}_{\beta}\right\rangle=0 .
$$

From this point, one employs the averaging theorem (Howes and Whitaker, 1985) and follows previous studies of two-phase flows (Whitaker, 1986, 1994) to obtain

$$
\frac{\partial \varepsilon_{\beta}}{\partial t}+\nabla \cdot\left\langle\mathbf{v}_{\beta}\right\rangle=0
$$

The analogous form of Equation (1.13) for the $\gamma$-phase is given by

$$
\frac{\partial \varepsilon_{\gamma}}{\partial t}+\nabla \cdot\left\langle\mathbf{v}_{\gamma}\right\rangle=0
$$

and for most two-phase flow problem the objective is to determine $\varepsilon_{\beta}$, and the superficial average velocities $\left\langle\mathbf{v}_{\beta}\right\rangle$ and $\left\langle\mathbf{v}_{\gamma}\right\rangle$.

\subsection{MOMENTUM EQUATION}

Forming the superficial volume average of Equation (1.6b) and applying the averaging theorem twice leads to

$$
0=-\nabla\left\langle p_{\beta}\right\rangle+\varepsilon_{\beta} \rho_{\beta} \mathbf{g}+\mu_{\beta} \nabla^{2}\left\langle\mathbf{v}_{\beta}\right\rangle+
$$




$$
\begin{aligned}
& +\mu_{\beta} \nabla \cdot\left[\frac{1}{\mathcal{V}} \int_{A_{\beta \sigma}} \mathbf{n}_{\beta \sigma} \mathbf{v}_{\beta} \mathrm{d} A+\frac{1}{\mathcal{V}} \int_{A_{\beta \gamma}} \mathbf{n}_{\beta \gamma} \mathbf{v}_{\beta} \mathrm{d} A\right]+ \\
& +\frac{1}{\mathcal{V}} \int_{A_{\beta \sigma}} \mathbf{n}_{\beta \sigma} \cdot\left(-p_{\beta} \mathbf{I}+\mu_{\beta} \nabla \mathbf{v}_{\beta}\right) \mathrm{d} A+ \\
& +\frac{1}{\mathcal{V}} \int_{A_{\beta \gamma}} \mathbf{n}_{\beta \gamma} \cdot\left(-p_{\beta} \mathbf{I}+\mu_{\beta} \nabla \mathbf{v}_{\beta}\right) \mathrm{d} A .
\end{aligned}
$$

Using the traditional length-scale arguments (Carbonell and Whitaker, 1984; Quintard and Whitaker, 1994) provides the simplification

$$
\mu_{\beta} \nabla \cdot\left[\frac{1}{\mathcal{V}} \int_{A_{\beta \gamma}} \mathbf{n}_{\beta \gamma} \mathbf{v}_{\beta} \mathrm{d} A\right] \ll \frac{1}{\mathcal{V}} \int_{A_{\beta \gamma}} \mathbf{n}_{\beta \gamma} \cdot\left(\mu_{\beta} \nabla \mathbf{v}_{\beta}\right) \mathrm{d} A
$$

and this can be used along with the no-slip condition given by Equation (1.6c) to simplify Equation (1.15) to the form

$$
\begin{aligned}
0= & -\nabla\left\langle p_{\beta}\right\rangle+\varepsilon_{\beta} \rho_{\beta} \mathbf{g}+\mu_{\beta} \nabla^{2}\left\langle\mathbf{v}_{\beta}\right\rangle+ \\
& +\frac{1}{\mathcal{V}} \int_{A_{\beta \sigma}+A_{\beta \gamma}} \mathbf{n}_{\beta} \cdot\left(-p_{\beta} \mathbf{I}+\mu_{\beta} \nabla \mathbf{v}_{\beta}\right) \mathrm{d} A .
\end{aligned}
$$

Here we have used $\mathbf{n}_{\beta}$ to represent both $\mathbf{n}_{\beta \sigma}$ and $\mathbf{n}_{\beta \gamma}$.

In general, superficial average transport equations, such as Equations (1.13) and (1.14), are preferred since each term in such equations represents some quantity per unit volume of the porous medium. However, in many cases intrinsic average variables are preferred and the pressure is one of these variables. Thus we use

$$
\left\langle p_{\beta}\right\rangle=\varepsilon_{\beta}\left\langle p_{\beta}\right\rangle^{\beta},
$$

in order to express Equation (1.17) as

$$
\begin{aligned}
0= & -\varepsilon_{\beta} \nabla\left\langle p_{\beta}\right\rangle^{\beta}-\left\langle p_{\beta}\right\rangle^{\beta} \nabla \varepsilon_{\beta}+\varepsilon_{\beta} \rho_{\beta} \mathbf{g}+\mu_{\beta} \nabla^{2}\left\langle\mathbf{v}_{\beta}\right\rangle+ \\
& +\frac{1}{\mathcal{V}} \int_{A_{\beta \sigma}+A_{\beta \gamma}} \mathbf{n}_{\beta} \cdot\left[-\mathbf{l} p_{\beta}+\mu_{\beta} \nabla \mathbf{v}_{\beta}\right] \mathrm{d} A .
\end{aligned}
$$

In order to remove the point values of the pressure and velocity from this volume averaged form of the Stokes' equations, we will make use of the following decompositions

$$
\begin{aligned}
& \boldsymbol{p}_{\beta}=\left\langle p_{\beta}\right\rangle^{\beta}+\tilde{p}_{\beta}, \\
& \mathbf{v}_{\beta}=\left\langle\mathbf{v}_{\beta}\right\rangle^{\beta}+\tilde{\mathbf{v}}_{\beta},
\end{aligned}
$$




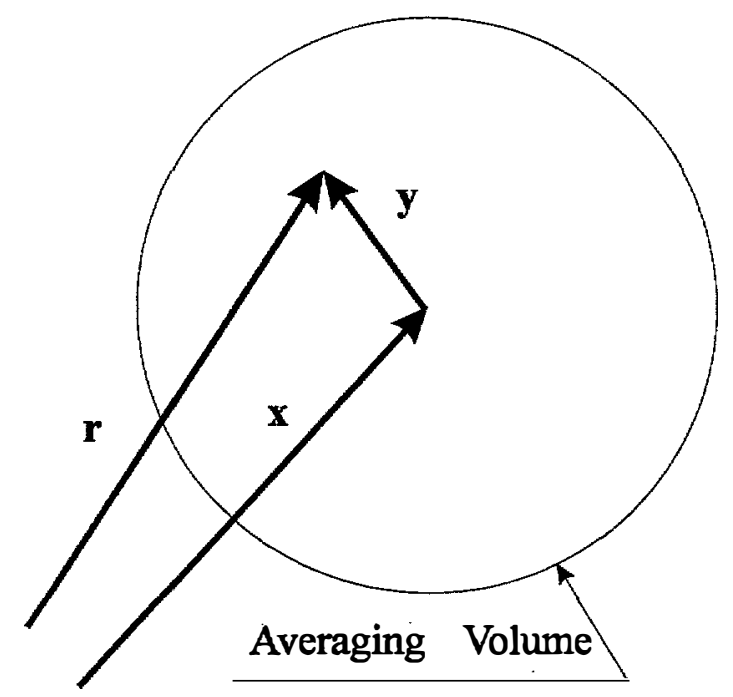

Figure 2. Position vectors associated with the averaging volume.

that allow us to express Equation (1.19) as

$$
\begin{aligned}
0= & -\varepsilon_{\beta} \nabla\left\langle p_{\beta}\right\rangle^{\beta}-\left\langle p_{\beta}\right\rangle^{\beta} \nabla \varepsilon_{\beta}+\varepsilon_{\beta} \rho_{\beta} \mathbf{g}+\mu_{\beta} \nabla^{2}\left\langle\mathbf{v}_{\beta}\right\rangle+ \\
& +\frac{1}{\mathcal{V}} \int_{A_{\beta \sigma}+A_{\beta \gamma}} \mathbf{n}_{\beta} \cdot\left[-\mathbf{I} \tilde{p}_{\beta}+\mu_{\beta} \nabla \tilde{\mathbf{v}}_{\beta}\right] \mathrm{d} A+ \\
& +\frac{1}{\mathcal{V}} \int_{A_{\beta \sigma}+A_{\beta \gamma}} \mathbf{n}_{\beta} \cdot\left[-\mathbf{I}\left\langle p_{\beta}\right\rangle^{\beta}+\mu_{\beta} \nabla\left\langle\mathbf{v}_{\beta}\right\rangle^{\beta}\right] \mathrm{d} A .
\end{aligned}
$$

This represents a nonlocal problem in terms of $\left\langle p_{\beta}\right\rangle^{\beta}$ and $\left\langle\mathbf{v}_{\beta}\right\rangle^{\beta}$ since these volume averaged quantities will be evaluated at points other than the centroid of the averaging volume which is located by the position vector $\mathbf{x}$ shown in Figure 2. We can avoid the difficulties associated with nonlocal problems (Quintard and Whitaker, 1990) if $\left\langle p_{\beta}\right\rangle^{\beta}$ and $\left\langle\mathbf{v}_{\beta}\right\rangle^{\beta}$ can be removed from the area integral in Equation (1.21). This matter has been discussed by Carbonell and Whitaker (1984) and more recently by Quintard and Whitaker (1994), and it is an acceptable simplification when the following length-scale constraints are satisfied

$$
\ell \ll r_{0} \quad r_{0}^{2} \ll L^{2}
$$

Here $\ell$ represents both $\ell_{\beta}$ and $\ell_{\gamma}$ and $L$ should be thought of as the smallest length scale associated with the volume averaged quantities. When the average quantities 
are removed from the area integral in Equation (1.21), and we make use of the lemma available from the averaging theorem

$$
\frac{1}{\mathcal{V}} \int_{A_{\beta \sigma}+A_{\beta \gamma}} \mathbf{n}_{\beta} \mathrm{d} A=-\nabla \varepsilon_{\beta}
$$

we obtain

$$
\begin{aligned}
0= & -\varepsilon_{\beta} \nabla\left\langle p_{\beta}\right\rangle^{\beta}+\varepsilon_{\beta} \rho_{\beta} \mathbf{g}+\underbrace{\mu_{\beta} \nabla^{2}\left\langle\mathbf{v}_{\beta}\right\rangle-\mu_{\beta} \nabla \varepsilon_{\beta} \cdot \nabla\left\langle\mathbf{v}_{\beta}\right\rangle^{\beta}}_{\text {Brinkman corrections }}+ \\
& +\frac{1}{\mathcal{V}} \int_{A_{\beta \sigma}+A_{\beta \gamma}} \mathbf{n}_{\beta} \cdot\left[-\mathbf{I} \tilde{p}_{\beta}+\mu_{\beta} \nabla \tilde{\mathbf{v}}_{\beta}\right] \mathbf{d} A .
\end{aligned}
$$

Here we have identified the first and second Brinkman corrections, both of which are negligible for typical two-phase flow problems. This means that we can divide Equation (1.24) by $\varepsilon_{\beta}$ so that our volume averaged momentum equation for the $\beta$-phase takes the form

$$
0=-\nabla\left\langle p_{\beta}\right\rangle^{\beta}+\rho_{\beta} \mathbf{g}+\frac{1}{V_{\beta}} \int_{A_{\beta \sigma}+A_{\beta \gamma}} \mathbf{n}_{\beta} \cdot\left[-\mathbf{I} \tilde{p}_{\beta}+\mu_{\beta} \nabla \tilde{\mathbf{v}}_{\beta}\right] \mathrm{d} A .
$$

For completeness we list the analogous form for the $\gamma$-phase as

$$
0=-\nabla\left\langle p_{\gamma}\right\rangle^{\gamma}+\rho_{\gamma} \mathbf{g}+\frac{1}{V_{\gamma}} \int_{A_{\gamma \sigma}+A_{\gamma \beta}} \mathbf{n}_{\gamma} \cdot\left[-\mathbf{l} \tilde{p}_{\gamma}+\mu_{\gamma} \nabla \tilde{\mathbf{v}}_{\gamma}\right] \mathrm{d} A
$$

These two results, along with Equations (1.13) and (1.14), represent the volume average transport equation associated with the boundary-value problem given by Equations (1.6). It is of some importance to note that Equations (1.25) and (1.26) are intrinsic average equations, thus each term has units of force per unit volume of the fluid phase while Equations (1.13) and (1.14) are superficial average equations and each term has units of volume of the $\beta$ or $\gamma$-phase per unit volume of the porous medium. The momentum equations have been arranged as intrinsic average equations because the intrinsic average pressures, $\left\langle p_{\beta}\right\rangle^{\beta}$ and $\left\langle p_{\gamma}\right\rangle^{\gamma}$, are required for interpretation of the capillary pressure and for the construction of boundary conditions that may involve the pressure.

To proceed from Equations (1.25) and (1.26) to the forms indicated by Equations (1.1) and (1.2) or to the forms indicated by Equations (1.4) and (1.5), we need to develop a closure problem for the spatial deviation pressures and velocities. Two extensive efforts have been directed toward the development of the closure problem (Whitaker, 1986, 1994) and in the next section we simply list the result and show how it can be used. 


\section{Closure Problem}

The closure problem associated with the two-phase flow process described by Equations (1.6) is given by (Whitaker, 1986)

$$
\begin{aligned}
& \nabla \cdot \tilde{\mathbf{v}}_{\beta}=0 \\
& 0=-\nabla \tilde{p}_{\beta}+\mu_{\beta} \nabla^{2} \tilde{\mathbf{v}}_{\beta}-\frac{1}{V_{\beta}} \int_{A_{\beta \sigma}+A_{\beta \gamma}} \mathbf{n}_{\beta} \cdot\left[-\mathbf{l} \tilde{p}_{\beta}+\mu_{\beta} \nabla \tilde{\mathbf{v}}_{\beta}\right] \mathrm{d} A,
\end{aligned}
$$

B.C.1 $\tilde{\mathbf{v}}_{\beta}=-\left\langle\mathbf{v}_{\beta}\right\rangle^{\beta}, \quad$ at $\mathcal{A}_{\beta \sigma}$,

B.C.2 $\tilde{\mathbf{v}}_{\beta}=\tilde{\mathbf{v}}_{\gamma}-\left(\left\langle\mathbf{v}_{\beta}\right\rangle^{\beta}-\left\langle\mathbf{v}_{\gamma}\right\rangle^{\gamma}\right)$, at $\mathcal{A}_{\beta \gamma}$,

B.C.3 $-\mathbf{n}_{\beta \gamma} \tilde{p}_{\beta}$

$$
\begin{aligned}
= & -\mathbf{n}_{\beta \gamma} \tilde{p}_{\gamma}-\mathbf{n}_{\beta \gamma} \cdot\left[\mu_{\beta}\left(\nabla \tilde{\mathbf{v}}_{\beta}+\nabla \tilde{\mathbf{v}}_{\beta}^{T}\right)-\mu_{\gamma}\left(\nabla \tilde{\mathbf{v}}_{\gamma}+\nabla \tilde{\mathbf{v}}_{\gamma}^{T}\right)\right]+ \\
& +\mathbf{n}_{\beta \gamma}\left(\left\langle p_{\beta}\right\rangle^{\beta}-\left\langle p_{\gamma}\right\rangle^{\gamma}\right)+2 \sigma H \mathbf{n}_{\beta \gamma}, \quad \text { at } \mathcal{A}_{\beta \gamma},
\end{aligned}
$$

B.C.4 $\tilde{\mathbf{v}}_{\gamma}=-\left\langle\mathbf{v}_{\gamma}\right\rangle^{\gamma}, \quad$ at $\mathcal{A}_{\gamma \sigma}$,

$$
\begin{aligned}
& 0=-\nabla \tilde{p}_{\gamma}+\mu_{\gamma} \nabla^{2} \tilde{\mathbf{v}}_{\gamma}-\frac{1}{V_{\gamma}} \int_{A_{\gamma \sigma}+A_{\gamma \beta}} \mathbf{n}_{\gamma} \cdot\left[-\mathbf{l} \tilde{p}_{\gamma}+\mu_{\gamma} \nabla \tilde{\mathbf{v}}_{\gamma}\right] \mathrm{d} A, \\
& \nabla \cdot \tilde{\mathbf{v}}_{\gamma}=0
\end{aligned}
$$

Averages: $\quad\left\langle\tilde{\mathbf{v}}_{\beta}\right\rangle^{\beta}=0, \quad\left\langle\tilde{\mathbf{v}}_{\gamma}\right\rangle^{\gamma}=0$.

Here we have discarded the boundary conditions imposed at $\mathcal{A}_{\beta e}$ and $\mathcal{A}_{\gamma e}$ with the idea that Equations (2.1) will lead us to a local problem for which spatially periodic boundary conditions will be used. For small values of the capillary number, $C a$, and small values of the Bond number, $B o$, one can argue that the mean curvature undergoes negligible variations within the averaging volume and this leads to (Torres, 1987)

$$
-\left(\left\langle p_{\beta}\right\rangle^{\beta}-\left\langle p_{\gamma}\right\rangle^{\gamma}\right)=2 \sigma H \quad C a \ll 1, \quad B o \ll 1 .
$$

This simplification has also been obtained by Auriault (1987) and it provides a closure problem in which there are only two nonhomogeneous terms, $\left\langle\mathbf{v}_{\beta}\right\rangle^{\beta}$ and $\left\langle\mathbf{v}_{\gamma}\right\rangle^{\gamma}$, and no effects of surface tension or gravity. Both surface tension and gravity can influence the location of the $\boldsymbol{\beta}-\gamma$ interface; however, neither effect appears in the closure problem when the capillary number and the Bond number are small compared to one. We refer to $\left\langle\mathbf{v}_{\beta}\right\rangle^{\beta}$ and $\left\langle\mathbf{v}_{\gamma}\right\rangle^{\gamma}$ as the source terms in the closure 
problem and they naturally lead to representations of the form

$$
\begin{aligned}
& \tilde{\mathbf{v}}_{\beta}=\mathbf{A}_{\beta \beta} \cdot\left\langle\mathbf{v}_{\beta}\right\rangle^{\beta}+\mathbf{A}_{\beta \gamma} \cdot\left\langle\mathbf{v}_{\gamma}\right\rangle^{\gamma}, \\
& \tilde{p}_{\beta}=\mu_{\beta}\left[\mathbf{a}_{\beta \beta} \cdot\left\langle\mathbf{v}_{\beta}\right\rangle^{\beta}+\mathbf{a}_{\beta \gamma} \cdot\left\langle\mathbf{v}_{\gamma}\right\rangle^{\gamma}\right], \\
& \tilde{\mathbf{v}}_{\gamma}=\mathbf{A}_{\gamma \beta} \cdot\left\langle\mathbf{v}_{\beta}\right\rangle^{\beta}+\mathbf{A}_{\gamma \gamma} \cdot\left\langle\mathbf{v}_{\gamma}\right\rangle^{\gamma}, \\
& \tilde{p}_{\gamma}=\mu_{\gamma}\left[\mathbf{a}_{\gamma \beta} \cdot\left\langle\mathbf{v}_{\beta}\right\rangle^{\beta}+\mathbf{a}_{\gamma \gamma} \cdot\left\langle\mathbf{v}_{\gamma}\right\rangle^{\gamma}\right] .
\end{aligned}
$$

The nomenclature used in this presentation has been arranged so that the first subscript always identifies the phase in which the function is defined, while the second subscript always indicates which velocity is being mapped onto a spatial deviation. For example, the vector $\mathbf{a}_{\beta \gamma}$ represents a function defined in the $\beta$-phase and it maps the velocity $\left\langle\mathbf{v}_{\gamma}\right\rangle^{\gamma}$ onto a spatial deviation variable. This spatial deviation variable must be a scalar defined in the $\beta$-phase, thus $\mathbf{a}_{\beta \gamma}$ is associated with a mapping of $\left\langle\mathbf{v}_{\gamma}\right\rangle^{\gamma}$ onto $\tilde{p}_{\beta}$. According to this convention, $\mathbf{A}_{\gamma \beta}$ is defined in the $\gamma$-phase and it must map $\left\langle\mathbf{v}_{\beta}\right\rangle^{\beta}$ onto $\tilde{\mathbf{v}}_{\gamma}$. The closure problem in terms of these mapping tensors and vectors (known as closure variables) is rather complex (Whitaker, 1986); however, a simple form can be extracted by a series of transformations (Whitaker, 1994). One begins by using Equations (2.3) in the volume averaged momentum equations given by Equations (1.25) and (1.26). For the $\beta$-phase this leads to

$$
\begin{aligned}
0= & -\nabla\left\langle p_{\beta}\right\rangle^{\beta}+\rho_{\beta} \mathbf{g}+\mu_{\beta} \varepsilon_{\beta}^{-1} \times \\
& \times\left\{\frac{1}{V_{\beta}} \int_{A_{\beta \sigma}+A_{\beta \gamma}} \mathbf{n}_{\beta} \cdot\left[-\mathbf{l} \mathbf{a}_{\beta \beta}+\nabla \mathbf{A}_{\beta \beta}\right]\right\} \cdot\left\langle\mathbf{v}_{\beta}\right\rangle+ \\
& +\mu_{\beta} \varepsilon_{\gamma}^{-1}\left\{\frac{1}{V_{\beta}} \int_{A_{\beta \sigma}+A_{\beta \gamma}} \mathbf{n}_{\beta} \cdot\left[-\mathbf{l a}_{\beta \gamma}+\nabla \mathbf{A}_{\beta \gamma}\right] \mathrm{d} A\right\} \cdot\left\langle\mathbf{v}_{\gamma}\right\rangle,
\end{aligned}
$$

while the $\gamma$-phase equation is given by

$$
\begin{aligned}
0= & -\nabla\left\langle p_{\gamma}\right\rangle^{\gamma}+\rho_{\gamma} \mathbf{g}+\mu_{\gamma} \varepsilon_{\beta}^{-1} \times \\
& \times\left\{\frac{1}{V_{\gamma}} \int_{A_{\gamma \sigma}+A_{\gamma \beta}} \mathbf{n}_{\gamma} \cdot\left[-\mathbf{l} \mathbf{a}_{\gamma \beta}+\nabla \mathbf{A}_{\gamma \beta}\right]\right\} \cdot\left\langle\mathbf{v}_{\beta}\right\rangle+ \\
& +\mu_{\gamma} \varepsilon_{\gamma}^{-1}\left\{\frac{1}{V_{\gamma}} \int_{A_{\gamma \sigma}+A_{\gamma \beta}} \mathbf{n}_{\gamma} \cdot\left[-\mathbf{l}_{\gamma \gamma}+\nabla \mathbf{A}_{\gamma \gamma}\right] \mathrm{d} A\right\} \cdot\left\langle\mathbf{v}_{\gamma}\right\rangle .
\end{aligned}
$$

Here it is important to note that we have arranged the intrinsic average momentum equations in a form that contains the superficial average velocity. This is done because the continuity equations (Equations (1.13) and (1.14)) take a simpler form when expressed in terms of superficial averages; however, one must remember that Equations (2.4) and (2.5) are still intrinsic average momentum equations. 
At this point we identify the integrals in terms of the permeability and viscous drag tensors according to

$$
\begin{aligned}
& \left\{\frac{1}{V_{\beta}} \int_{A_{\beta \sigma}+A_{\beta \gamma}} \mathbf{n}_{\beta} \cdot\left[-\mathbf{l} \mathbf{a}_{\beta \beta}+\nabla \mathbf{A}_{\beta \beta}\right]\right\}=-\varepsilon_{\beta} \mathbf{K}_{\beta}^{-1}, \\
& \left\{\frac{1}{V_{\beta}} \int_{A_{\beta \sigma}+A_{\beta \gamma}} \mathbf{n}_{\beta} \cdot\left[-\mathbf{l} \mathbf{a}_{\beta \gamma}+\nabla \mathbf{A}_{\beta \gamma}\right] \mathrm{d} A\right\}=\varepsilon_{\gamma} \mathbf{K}_{\beta}^{-1} \cdot \mathbf{K}_{\beta \gamma}, \\
& \left\{\frac{1}{V_{\gamma}} \int_{A_{\gamma \sigma}+A_{\gamma \beta}} \mathbf{n}_{\gamma} \cdot\left[-\mathbf{l} \mathbf{a}_{\gamma \beta}+\nabla \mathbf{A}_{\gamma \beta}\right] \mathrm{d} A\right\}=\varepsilon_{\beta} \mathbf{K}_{\gamma}^{-1} \cdot \mathbf{K}_{\gamma \beta}, \\
& \left\{\frac{1}{V_{\gamma}} \int_{A_{\gamma \sigma}+A_{\gamma \beta}} \mathbf{n}_{\gamma} \cdot\left[-\mathbf{l} \mathbf{a}_{\gamma \gamma}+\nabla \mathbf{A}_{\gamma \gamma}\right] \mathrm{d} A\right\}=\varepsilon_{\gamma} \mathbf{K}_{\gamma}^{-1} .
\end{aligned}
$$

Use of these results in Equations (2.4) and (2.5) gives the closed form of the momentum equations indicated by Equations (1.1) and (1.2), i.e., the form containing the viscous drag tensors. In turn, one can use Equations (1.1) and (1.2) to obtain Equations (1.4) and (1.5), i.e., the form containing the permeability tensors which are defined by

$$
\begin{aligned}
& \mathbf{K}_{\beta \beta}^{*}=\left[\mathbf{I}-\mathbf{K}_{\beta \gamma} \cdot \mathbf{K}_{\gamma \beta}\right]^{-1} \cdot \mathbf{K}_{\beta}, \\
& \mathbf{K}_{\beta \gamma}^{*}=\left[\mathbf{I}-\mathbf{K}_{\beta \gamma} \cdot \mathbf{K}_{\gamma \beta}\right]^{-1} \cdot\left(\mathbf{K}_{\beta \gamma} \cdot \mathbf{K}_{\gamma}\right), \\
& \mathbf{K}_{\gamma \beta}^{*}=\left[\mathbf{I}-\mathbf{K}_{\gamma \beta} \cdot \mathbf{K}_{\beta \gamma}\right]^{-1} \cdot\left(\mathbf{K}_{\gamma \beta} \cdot \mathbf{K}_{\beta}\right), \\
& \mathbf{K}_{\gamma \gamma}^{*}=\left[\mathbf{I}-\mathbf{K}_{\gamma \beta} \cdot \mathbf{K}_{\beta \gamma}\right]^{-1} \cdot \mathbf{K}_{\gamma} .
\end{aligned}
$$

Both sets of tensors can be extracted from essentially the same closure problem and the development of the closure problem, in terms of closure variables, begins by substituting the representations given by Equations (2.3) into the closure problem represented by Equations (2.1). This leads to a pair of complex closure problems (see Problems I and II of Whitaker, 1994); however, they can be simplified by the following transformations

$$
\begin{aligned}
& \mathbf{A}_{\beta \beta}=-\mathbf{I}-\varepsilon_{\beta}\left[\mathbf{D}_{\beta \beta}^{0} \cdot \mathbf{K}_{\beta}^{-1}-\mathbf{D}_{\beta \gamma}^{0} \cdot\left(\mathbf{K}_{\gamma}^{-1} \cdot \mathbf{K}_{\gamma \beta}\right)\right], \\
& \mathbf{a}_{\beta \beta}=-\varepsilon_{\beta}\left[\mathbf{d}_{\beta \beta}^{0} \cdot \mathbf{K}_{\beta}^{-1}-\mathbf{d}_{\beta \gamma}^{0} \cdot\left(\mathbf{K}_{\gamma}^{-1} \cdot \mathbf{K}_{\gamma \beta}\right)\right], \\
& \mathbf{A}_{\gamma \beta}=-\varepsilon_{\beta}\left[\mathbf{D}_{\gamma \beta}^{0} \cdot \mathbf{K}_{\beta}^{-1}-\mathbf{D}_{\gamma \gamma}^{0} \cdot\left(\mathbf{K}_{\gamma}^{-1} \cdot \mathbf{K}_{\gamma \beta}\right)\right], \\
& \mathbf{a}_{\gamma \beta}=-\varepsilon_{\beta}\left[\mathbf{d}_{\gamma \beta}^{0} \cdot \mathbf{K}_{\beta}^{-1}-\mathbf{d}_{\gamma \gamma}^{0} \cdot\left(\mathbf{K}_{\gamma}^{-1} \cdot \mathbf{K}_{\gamma \beta}\right)\right],
\end{aligned}
$$




$$
\begin{aligned}
& \mathbf{A}_{\gamma \gamma}=-\mathbf{I}-\varepsilon_{\gamma}\left[\mathbf{D}_{\gamma \gamma}^{0} \cdot \mathbf{K}_{\gamma}^{-1}-\mathbf{D}_{\gamma \beta}^{0} \cdot\left(\mathbf{K}_{\beta}^{-1} \cdot \mathbf{K}_{\beta \gamma}\right)\right], \\
& \mathbf{a}_{\gamma \gamma}=-\varepsilon_{\gamma}\left[\mathbf{d}_{\gamma \gamma}^{0} \cdot \mathbf{K}_{\gamma}^{-1}-\mathbf{d}_{\gamma \beta}^{0} \cdot\left(\mathbf{K}_{\beta}^{-1} \cdot \mathbf{K}_{\beta \gamma}\right)\right], \\
& \mathbf{A}_{\beta \gamma}=-\varepsilon_{\gamma}\left[\mathbf{D}_{\beta \gamma}^{0} \cdot \mathbf{K}_{\gamma}^{-1}-\mathbf{D}_{\beta \beta}^{0} \cdot\left(\mathbf{K}_{\beta}^{-1} \cdot \mathbf{K}_{\beta \gamma}\right)\right], \\
& \mathbf{a}_{\beta \gamma}=-\varepsilon_{\gamma}\left[\mathbf{d}_{\beta \gamma}^{0} \cdot \mathbf{K}_{\gamma}^{-1}-\mathbf{d}_{\beta \beta}^{0} \cdot\left(\mathbf{K}_{\beta}^{-1} \cdot \mathbf{K}_{\beta \gamma}\right)\right] .
\end{aligned}
$$

These transformations lead to Problems I(b) and I( $\left.\mathrm{b}^{\prime}\right)$ of Whitaker (1994); however, we have made extensive use of Equations (4.26) and (4.27) of Whitaker (1994) in order to arrive at a nomenclature that is simpler than what one finds in the original development. Use of Equations (2.8) and (2.9) provides two relatively simple closure problems. The first problem has the appearance of a Stokes flow problem with the single nonhomogeneous term being the unit tensor in Equation (2.10b).

Problem I(b):

$$
\begin{aligned}
& \nabla \cdot \mathbf{D}_{\beta \beta}^{0}=0, \quad \text { in the } \beta \text {-phase, } \\
& -\nabla \mathbf{d}_{\beta \beta}^{0}+\nabla^{2} \mathbf{D}_{\beta \beta}^{0}=\mathbf{I}, \quad \text { in the } \beta \text {-phase, }
\end{aligned}
$$

B.C.1 $\quad \mathbf{D}_{\beta \beta}^{0}=0, \quad$ at $A_{\beta \sigma}$,

B.C. $\quad \mathbf{D}_{\beta \beta}^{0}=\mathbf{D}_{\gamma \beta}^{0}, \quad$ at $A_{\beta \gamma}$,

B.C.3 $\mu_{\beta} \mathbf{n}_{\beta \gamma} \cdot\left[-\mathbf{I d}_{\beta \beta}^{0}+\left(\nabla \mathbf{D}_{\beta \beta}^{0}+\nabla \mathbf{D}_{\beta \beta}^{0 T}\right)\right]=$

$$
=\mu_{\gamma} \mathbf{n}_{\beta \gamma} \cdot\left[-\mathbf{I d}_{\gamma \beta}^{0}+\left(\nabla \mathbf{D}_{\gamma \beta}^{0}+\nabla \mathbf{D}_{\gamma \beta}^{0 T}\right)\right], \quad \text { at } A_{\beta \gamma},
$$

B.C. $4 \quad \mathbf{D}_{\gamma \beta}^{0}=0, \quad$ at $A_{\gamma \sigma}$,

$$
-\nabla \mathbf{d}_{\gamma \beta}^{0}+\nabla^{2} \mathbf{D}_{\gamma \beta}^{0}=0, \quad \text { in the } \gamma \text {-phase, }
$$

$\nabla \cdot \mathbf{D}_{\gamma \beta}^{0}=0, \quad$ in the $\gamma$-phase,

Periodicity: $\quad \mathbf{d}_{\beta \beta}^{0}\left(\mathbf{r}+\ell_{i}\right)=\mathbf{d}_{\beta \beta}^{0}(\mathbf{r}), \quad \mathbf{D}_{\beta \beta}^{0}\left(\mathbf{r}+\ell_{i}\right)=\mathbf{D}_{\beta \beta}^{0}(\mathbf{r})$,

$$
\begin{aligned}
& i=1,2,3, \\
& \mathbf{d}_{\gamma \beta}^{0}\left(\mathbf{r}+\ell_{i}\right)=\mathbf{d}_{\gamma \beta}^{0}(\mathbf{r}), \quad \mathbf{D}_{\gamma \beta}^{0}\left(\mathbf{r}+\ell_{i}\right)=\mathbf{D}_{\gamma \beta}^{0}(\mathbf{r}), \\
& i=1,2,3,
\end{aligned}
$$

Average: $\quad\left\langle\mathbf{D}_{\beta \beta}^{0}\right\rangle^{\beta}=-\varepsilon_{\beta}^{-1} \mathbf{K}_{\beta \beta}^{*}, \quad\left\langle\mathbf{D}_{\gamma \beta}^{0}\right\rangle^{\gamma}=-\varepsilon_{\gamma}^{-1} \mathbf{K}_{\gamma \beta}^{*}$. 
Here we see that two of the desired permeability tensors are given by the averages of the tensor fields as indicated in Equations (2.10k). The second problem is almost identical to the first with the exception that the nonhomogeneous term now appears in the $\gamma$-phase transport equation instead of the equivalent $\beta$-phase equation.

Problem $\mathrm{I}\left(\mathrm{b}^{\prime}\right)$

$$
\begin{aligned}
& \nabla \cdot \mathbf{D}_{\beta \gamma}^{0}=0, \quad \text { in the } \beta \text {-phase, } \\
& -\nabla \mathbf{d}_{\beta \gamma}^{0}+\nabla^{2} \mathbf{D}_{\beta \gamma}^{0}=0, \quad \text { in the } \beta \text {-phase, }
\end{aligned}
$$

B.C.1 $\quad \mathbf{D}_{\beta \gamma}^{0}=0$, at $A_{\beta \sigma}$,

B.C.2 $\quad \mathbf{D}_{\beta \gamma}^{0}=\mathbf{D}_{\gamma \gamma}^{0}, \quad$ at $A_{\beta \gamma}$,

B.C.3 $\mu_{\beta} \mathbf{n}_{\beta \gamma} \cdot\left[-\mathbf{I d}_{\beta \gamma}^{0}+\left(\nabla \mathbf{D}_{\beta \gamma}^{0}+\nabla \mathbf{D}_{\beta \gamma}^{0 T}\right)\right]=$

$$
=\mu_{\gamma} \mathbf{n}_{\beta \gamma} \cdot\left[-\mathbf{l} \mathbf{d}_{\gamma \gamma}^{0}+\left(\nabla \mathbf{D}_{\gamma \gamma}^{0}+\nabla \mathbf{D}_{\gamma \gamma}^{0 T}\right)\right], \quad \text { at } A_{\beta \gamma},
$$

B.C.4 $\mathbf{D}_{\gamma \gamma}^{0}=0$, at $A_{\gamma \sigma}$,

$$
-\nabla \mathbf{d}_{\gamma \gamma}^{0}+\nabla^{2} \mathbf{D}_{\gamma \gamma}^{0}=\mathbf{I}, \quad \text { in the } \gamma \text {-phase, }
$$

$\nabla \cdot \mathbf{D}_{\gamma \gamma}^{0}=0, \quad$ in the $\gamma$-phase,

Periodicity: $\quad \mathbf{d}_{\beta \gamma}^{0}\left(\mathbf{r}+\ell_{i}\right)=\mathbf{d}_{\beta \gamma}^{0}(\mathbf{r}), \quad \mathbf{D}_{\beta \gamma}^{0}\left(\mathbf{r}+\ell_{i}\right)=\mathbf{D}_{\beta \gamma}^{0}(\mathbf{r})$,

$$
\begin{aligned}
& i=1,2,3, \\
& \mathbf{d}_{\gamma \gamma}^{0}\left(\mathbf{r}+\ell_{i}\right)=\mathbf{d}_{\gamma \gamma}^{0}(\mathbf{r}), \quad \mathbf{D}_{\gamma \gamma}^{0}\left(\mathbf{r}+\ell_{i}\right)=\mathbf{D}_{\gamma \gamma}^{0}(\mathbf{r}), \\
& i=1,2,3,
\end{aligned}
$$

Average: $\quad\left\langle\mathbf{D}_{\beta \gamma}^{0}\right\rangle^{\beta}=-\varepsilon_{\beta}^{-1} \mathbf{K}_{\beta \gamma}^{*}, \quad\left\langle\mathbf{D}_{\gamma \gamma}^{0}\right\rangle^{\gamma}=-\varepsilon_{\gamma}^{-1} \mathbf{K}_{\gamma \gamma}^{*}$.

This problem produces the third and fourth permeability tensors that are necessary to complete our description of the momentum equations given by Equations (1.4) and (1.5). In these two closure problems we have used spatially periodic conditions that essentially replace the boundary conditions imposed at $\mathcal{A}_{\beta e}$ and $\mathcal{A}_{\gamma e}$ in the original physical problem given by Equations (1.6).

In these closure problems we see two Stokes-like boundary value problems that can be solved with any routine capable of solving Stokes' equations for a two-phase flow. Once the tensorial fields are determined, the averages can be computed in order to determine the four permeability tensors that appear in Equations (1.4) and (1.5). The tensors that appear in Equations (1.1) and (1.2) can also be calculated on 
the basis of Equations (2.10) and (2.11); however, the form of the volume averaged momentum equations given by Equations (1.4) and (1.5) is preferred, thus we seek the permeability tensors given by Equations $(2.10 \mathrm{k})$ and $(2.11 \mathrm{k})$. The method of solving Equations (2.10) and (2.11) using numerical routines for Stokes flow in two-phase systems is outlined in the following section.

\section{Determination of Permeabilities}

In order to illustrate how the two closure problems given by Equations (2.10) and (2.11) can be solved, we use the arbitrary unit vector $\mathbf{e}_{0}$ to define the following pressure-like variables

$$
P_{\beta}^{0}=\mathbf{d}_{\beta \beta}^{0} \cdot \mathbf{e}_{0}, \quad P_{\gamma}^{0}=\mathbf{d}_{\gamma \beta}^{0} \cdot \mathbf{e}_{0}
$$

and the following velocity-like variables

$$
\mathbf{U}_{\beta}^{0}=\mathbf{D}_{\beta \beta}^{0} \cdot \mathbf{e}_{0}, \quad \mathbf{U}_{\gamma}^{0}=\mathbf{D}_{\gamma \beta}^{0} \cdot \mathbf{e}_{0} .
$$

We then form the scalar product of Equations (2.10) with $\mathbf{e}_{0}$ to obtain the following boundary value problem

Problem I(c):

$$
\begin{aligned}
& \nabla \cdot \mathbf{U}_{\beta}^{0}=0, \quad \text { in the } \beta \text {-phase, } \\
& 0=-\nabla P_{\beta}^{0}-\mathbf{e}_{0}+\nabla^{2} \mathbf{U}_{\beta}^{0}, \quad \text { in the } \beta \text {-phase, }
\end{aligned}
$$

B.C.3 $\mu_{\beta} \mathbf{n}_{\beta \gamma} \cdot\left[-\mathbf{I} P_{\beta}^{0}+\left(\nabla \mathbf{U}_{\beta}^{0}+\nabla \mathbf{U}_{\beta}^{0 T}\right)\right]=$

$$
=\mu_{\gamma} \mathbf{n}_{\beta \gamma} \cdot\left[-\mid P_{\gamma}^{0}+\left(\nabla \mathbf{U}_{\gamma}^{0}+\nabla \mathbf{U}_{\gamma}^{0 T}\right)\right], \quad \text { at } A_{\beta \gamma},
$$

B.C.4 $\mathbf{U}_{\gamma}^{0}=0$, at $A_{\gamma \sigma}$,

$$
\begin{aligned}
& 0=-\nabla P_{\gamma}^{0}+\nabla^{2} \mathbf{U}_{\gamma}^{0}, \quad \text { in the } \gamma \text {-phase, } \\
& \nabla \cdot \mathbf{U}_{\gamma}^{0}=0, \quad \text { in the } \gamma \text {-phase, }
\end{aligned}
$$

Periodicity: $\quad P_{\beta}^{0}\left(\mathbf{r}+\ell_{i}\right)=P_{\beta}^{0}(\mathbf{r}), \quad \mathbf{U}_{\beta}^{0}\left(\mathbf{r}+\ell_{i}\right)=\mathbf{U}_{\beta}^{0}(\mathbf{r})$,

$$
\begin{aligned}
& i=1,2,3, \\
& P_{\gamma}^{0}\left(\mathbf{r}+\ell_{i}\right)=P_{\gamma}^{0}(\mathbf{r}), \quad \mathbf{U}_{\gamma}^{0}\left(\mathbf{r}+\ell_{i}\right)=\mathbf{U}_{\gamma}^{0}(\mathbf{r}), \\
& i=1,2,3,
\end{aligned}
$$

Average: $\quad\left\langle\mathbf{U}_{\beta}^{0}\right\rangle^{\beta}=-\varepsilon_{\beta}^{-1} \mathbf{K}_{\beta \beta}^{*} \cdot \mathbf{e}_{0}, \quad\left\langle\mathbf{U}_{\gamma}^{0}\right\rangle^{\gamma}=-\varepsilon_{\gamma}^{-1} \mathbf{K}_{\gamma \beta}^{*} \cdot \mathbf{e}_{0}$. 
By choosing the unit vector $\mathbf{e}_{0}$ to be the unit base vectors $\mathbf{i}, \mathbf{j}$, and $\mathbf{k}$, we can use Equations (3.2) to calculate all the components of the two permeability tensors, $\mathbf{K}_{\beta \beta}^{*}$ and $\mathbf{K}_{\gamma \beta}^{*}$. Clearly the above boundary value problem has the same form as the original physical problem described by Equations (1.6) with the exception that the effect of surface tension is not present in Equations (3.2) and the boundary conditions at $\mathcal{A}_{\beta e}$ and $\mathcal{A}_{\gamma e}$ have been replaced by spatially periodic conditions. In Equation (3.2b), the unit vector $\mathbf{e}_{0}$ plays the role of gravity while 'gravitational effects' are absent from Equation (3.2g). In addition to solving Equations (3.2), one must solve the analogous closure problem derived from Equations (2.10), and in that case the unit vector will appear in the $\gamma$-phase 'momentum equation'. To be precise about this, we express this second boundary value problem as

Problem $\mathrm{I}\left(\mathrm{c}^{\prime}\right)$

$$
\begin{aligned}
& \nabla \cdot \mathbf{U}_{\beta}^{1}=0, \quad \text { in the } \beta \text {-phase, } \\
& 0=-\nabla P_{\beta}^{1}+\nabla^{2} \mathbf{U}_{\beta}^{1}, \quad \text { in the } \beta \text {-phase, }
\end{aligned}
$$

B.C.1 $\mathbf{U}_{\beta}^{1}=0, \quad$ at $A_{\beta \sigma}$,

B.C.2 $\quad \mathbf{U}_{\beta}^{1}=\mathbf{U}_{\gamma}^{1}, \quad$ at $A_{\beta \gamma}$,

B.C.3 $\mu_{\beta} \mathbf{n}_{\beta \gamma} \cdot\left[-I P_{\beta}^{1}+\left(\nabla \mathbf{U}_{\beta}^{1}+\nabla \mathbf{U}_{\beta}^{1 T}\right)\right]=$

$$
=\mu_{\gamma} \mathbf{n}_{\beta \gamma} \cdot\left[-I P_{\gamma}^{1}+\left(\nabla \mathbf{U}_{\gamma}^{1}+\nabla \mathbf{U}_{\gamma}^{1 T}\right)\right], \quad \text { at } A_{\beta \gamma},
$$

B.C.4 $\mathbf{U}_{\gamma}^{1}=0, \quad$ at $A_{\gamma \sigma}$,

$0=-\nabla P_{\gamma}^{1}-\mathbf{e}_{1}+\nabla^{2} \mathbf{U}_{\gamma}^{1}, \quad$ in the $\gamma$-phase,

$\nabla \cdot \mathbf{U}_{\gamma}^{1}=0, \quad$ in the $\gamma$-phase,

Periodicity: $\quad P_{\beta}^{1}\left(\mathbf{r}+\ell_{i}\right)=P_{\beta}^{1}(\mathbf{r}), \quad \mathbf{U}_{\beta}^{1}\left(\mathbf{r}+\ell_{i}\right)=\mathbf{U}_{\beta}^{1}(\mathbf{r})$,

$$
\begin{aligned}
& i=1,2,3, \\
& P_{\gamma}^{1}\left(\mathbf{r}+\ell_{i}\right)=P_{\gamma}^{1}(\mathbf{r}), \quad \mathbf{U}_{\gamma}^{1}\left(\mathbf{r}+\ell_{i}\right)=\mathbf{U}_{\gamma}^{1}(\mathbf{r}), \\
& i=1,2,3,
\end{aligned}
$$

Average: $\quad\left\langle\mathbf{U}_{\beta}^{1}\right\rangle^{\beta}=-\varepsilon_{\beta}^{-1} \mathbf{K}_{\beta \gamma}^{*} \cdot \mathbf{e}_{1}, \quad\left\langle\mathbf{U}_{\gamma}^{1}\right\rangle^{\gamma}=-\varepsilon_{\gamma}^{-1} \mathbf{K}_{\gamma \gamma}^{*} \cdot \mathbf{e}_{1}$.

Here we have used definitions analogous to those given by Equations (3.1) and for completeness we list the pressure-like variables and velocity-like variables as

$$
\begin{array}{ll}
P_{\beta}^{1}=\mathbf{d}_{\beta \gamma}^{0} \cdot \mathbf{e}_{1}, & P_{\gamma}^{1}=\mathbf{d}_{\gamma \gamma}^{0} \cdot \mathbf{e}_{1}, \\
\mathbf{U}_{\beta}^{1}=\mathbf{D}_{\beta \gamma}^{0} \cdot \mathbf{e}_{1}, & \mathbf{U}_{\gamma}^{1}=\mathbf{D}_{\gamma \gamma}^{0} \cdot \mathbf{e}_{1},
\end{array}
$$


Once again, we note that the arbitrary unit vector $\mathbf{e}_{1}$ can be taken to be the unit base vectors $\mathbf{i}, \mathbf{j}$, and $\mathbf{k}$ in order to calculate all the components of the two permeability tensors, $\mathbf{K}_{\beta \gamma}^{*}$ and $\mathbf{K}_{\gamma \gamma}^{*}$.

It is necessary to solve both closure problems in order to determine how the total drag will be distributed between the two pressure gradients that appear in Equations (1.4) and (1.5). The solution of the two closure problems must be preceded by the solution of the physical problem described by Equations (1.6) in order to locate the position of the $\beta-\gamma$ interface. In those problems for which the position of the interface can be specified a priori, one can proceed directly to the solution of the closure problems and the determination of the permeability tensors. However, it should be emphasized that no closure problems exist that lead to a complete determination of the position of the interface.

\subsection{NEGLIGIBLE COUPLING}

If one believes that the coupling permeability tensors are negligible, i.e.,

$$
\mathbf{K}_{\beta \beta}^{*} \gg \mathbf{K}_{\beta \gamma}^{*}, \quad \mathbf{K}_{\gamma \gamma}^{*} \gg \mathbf{K}_{\gamma \beta}^{*},
$$

only a single closure problem needs to be solved. In this case we use $\mathbf{e}_{0}=\mathbf{e}_{1}=\mathbf{e}$ and we form the sum of Problems (Ic) and (Ic') to obtain

Problem I(d)

$$
\begin{aligned}
& \nabla \cdot \mathbf{U}_{\beta}=0, \quad \text { in the } \beta \text {-phase, } \\
& 0=-\nabla P_{\beta}-\mathbf{e}+\nabla^{2} \mathbf{U}_{\beta}, \quad \text { in the } \beta \text {-phase, }
\end{aligned}
$$

B.C.1 $\mathbf{U}_{\beta}=0$, at $A_{\beta \sigma}$,

B.C.2 $\mathbf{U}_{\beta}=\mathbf{U}_{\gamma}, \quad$ at $A_{\beta \gamma}$,

B.C.3 $\mu_{\beta} \mathbf{n}_{\beta \gamma} \cdot\left[-\mathbf{I} P_{\beta}+\left(\nabla \mathbf{U}_{\beta}+\nabla \mathbf{U}_{\beta}^{T}\right)\right]=$

$$
=\mu_{\gamma} \mathbf{n}_{\beta \gamma} \cdot\left[-I P_{\gamma}+\left(\nabla \mathbf{U}_{\gamma}+\nabla \mathbf{U}_{\gamma}^{T}\right)\right], \quad \text { at } A_{\beta \gamma},
$$

B.C.4 $\mathbf{U}_{\gamma}=0$, at $A_{\gamma \sigma}$,

$$
\begin{aligned}
& 0=-\nabla P_{\gamma}-\mathbf{e}+\nabla^{2} \mathbf{U}_{\gamma}, \quad \text { in the } \gamma \text {-phase, } \\
& \nabla \cdot \mathbf{U}_{\gamma}=0, \quad \text { in the } \gamma \text {-phase, }
\end{aligned}
$$

Periodicity: $\quad P_{\beta}\left(\mathbf{r}+\ell_{i}\right)=P_{\beta}(\mathbf{r}), \quad \mathbf{U}_{\beta}\left(\mathbf{r}+\ell_{i}\right)=\mathbf{U}_{\beta}(\mathbf{r})$,

$$
\begin{aligned}
& i=1,2,3, \\
& P_{\gamma}\left(\mathbf{r}+\ell_{i}\right)=P_{\gamma}(\mathbf{r}), \quad \mathbf{U}_{\gamma}\left(\mathbf{r}+\ell_{i}\right)=\mathbf{U}_{\gamma}(\mathbf{r}), \\
& i=1,2,3,
\end{aligned}
$$




$$
\text { Average: } \quad \begin{aligned}
\left\langle\mathbf{U}_{\beta}\right\rangle^{\beta} & =-\varepsilon_{\beta}^{-1}\left(\mathbf{K}_{\beta \beta}^{*}+\mathbf{K}_{\beta \gamma}^{*}\right) \cdot \mathbf{e}, \\
& \left\langle\mathbf{U}_{\gamma}\right\rangle^{\gamma}=-\varepsilon_{\gamma}^{-1}\left(\mathbf{K}_{\gamma \beta}^{*}+\mathbf{K}_{\gamma \gamma}^{*}\right) \cdot \mathbf{e} .
\end{aligned}
$$

Here we have used the obvious definitions given by

$$
P_{\beta}=P_{\beta}^{0}+P_{\beta}^{1}, \quad \mathbf{U}_{\beta}=\mathbf{U}_{\beta}^{0}+\mathbf{U}_{\beta}^{1}
$$

and on the basis of the assumption indicated by Equations (3.5) we see that this closure problem can be used to determine the two significant permeability tensors according to

$$
\left\langle\mathbf{U}_{\beta}\right\rangle^{\beta}=-\varepsilon_{\beta}^{-1} \mathbf{K}_{\beta \beta}^{*} \cdot \mathbf{e}, \quad\left\langle\mathbf{U}_{\gamma}\right\rangle^{\gamma}=-\varepsilon_{\gamma}^{-1} \mathbf{K}_{\gamma \gamma}^{*} \cdot \mathbf{e} .
$$

The boundary value problem given by Equations (3.6) looks very much like the original physical problem given by Equations (1.6) and this suggests a more direct route to the closure problem.

\subsection{PHYSICAL PROBLEM}

The similarity between the closure problem and the physical problem can be made clear if we utilize the decomposition of the pressure indicated by Equation (1.20a) for both phases and then make use of Equation (2.2) to eliminate capillary effects. This leads us to the following version of Equations (1.6).

Physical Problem (a)

$$
\begin{aligned}
& \nabla \cdot \mathbf{v}_{\beta}=0, \quad \text { in the } \beta \text {-phase, } \\
& 0=-\nabla \tilde{p}_{\beta}-\Omega_{\beta}+\mu_{\beta} \nabla^{2} \mathbf{v}_{\beta}, \quad \text { in the } \beta \text {-phase, }
\end{aligned}
$$

B.C.1 $\quad \mathbf{v}_{\beta}=0, \quad$ at $\mathcal{A}_{\beta \sigma}$,

B.C.2 $\mathbf{v}_{\beta}=\mathbf{v}_{\gamma}, \quad$ at $\mathcal{A}_{\beta \gamma}$,

B.C.3 $-\mathbf{n}_{\boldsymbol{\beta} \gamma} \tilde{p}_{\beta}+\mathbf{n}_{\beta \gamma \gamma} \cdot\left[\mu_{\beta}\left(\nabla \mathbf{v}_{\beta}+\nabla \mathbf{v}_{\beta}^{T}\right)\right]=$

$$
=-\mathbf{n}_{\beta \gamma} \tilde{p}_{\gamma}+\mathbf{n}_{\beta \gamma} \cdot\left[\mu_{\gamma}\left(\nabla \mathbf{v}_{\gamma}+\nabla \mathbf{v}_{\gamma}^{T}\right)\right], \quad \text { at } \mathcal{A}_{\beta \gamma},
$$

B.C.4 $\mathbf{v}_{\gamma}=0$, at $\mathcal{A}_{\gamma \sigma}$,

$0=-\nabla \tilde{p}_{\gamma}-\Omega_{\gamma}+\mu_{\gamma} \nabla^{2} \mathbf{v}_{\gamma}, \quad$ in the $\gamma$-phase,

$\nabla \cdot \mathbf{v}_{\gamma}=0, \quad$ in the $\gamma$-phase,

B.C.5 $\mathbf{v}_{\beta}=\mathbf{f}(t)$ at $\mathcal{A}_{\beta e}$,

B.C.6 $\mathbf{v}_{\gamma}=\mathbf{g}(t), \quad$ at $\mathcal{A}_{\gamma e}$. 
Here we have used the definitions

$$
\Omega_{\beta}=\nabla\left\langle p_{\beta}\right\rangle^{\beta}-\rho_{\beta} \mathbf{g}, \quad \Omega_{\gamma}=\nabla\left\langle p_{\gamma}\right\rangle^{\gamma}-\rho_{\gamma} \mathbf{g}
$$

and we will assume that variations of the vectors $\Omega_{\beta}$ and $\Omega_{\gamma}$ can be neglected within the averaging volume. In this form, $\Omega_{\beta}$ and $\Omega_{\gamma}$ play the role of sources in the boundary value problem for the velocity $\mathbf{v}$ and the pressure deviation $\tilde{p}$, and this encourages us to express the dependent variables as

$$
\begin{aligned}
& \mathbf{v}_{\beta}=\frac{\mathbf{M}_{\beta \beta}}{\mu_{\beta}} \cdot \Omega_{\beta}+\frac{\mathbf{M}_{\beta \gamma}}{\mu_{\gamma}} \cdot \Omega_{\gamma}, \\
& \tilde{p}_{\beta}=\mathbf{m}_{\beta \beta} \cdot \Omega_{\beta}+\mathbf{m}_{\beta \gamma} \cdot \Omega_{\gamma}\left(\frac{\mu_{\beta}}{\mu_{\gamma}}\right), \\
& \mathbf{v}_{\gamma}=\frac{\mathbf{M}_{\gamma \beta}}{\mu_{\beta}} \cdot \Omega_{\beta}+\frac{\mathbf{M}_{\gamma \gamma}}{\mu_{\gamma}} \cdot \Omega_{\gamma}, \\
& \tilde{p}_{\gamma}=\mathbf{m}_{\gamma \beta} \cdot \Omega_{\beta}\left(\frac{\mu_{\gamma}}{\mu_{\beta}}\right)+\mathbf{m}_{\gamma \gamma} \cdot \Omega_{\gamma} .
\end{aligned}
$$

These representations are similar in form to those given by Equations (2.3); however, in this case we have a mixture of point variables and spatial deviation variables. In making use of these representations we have assumed that the boundary conditions for the point velocities, $\mathbf{v}_{\beta}$ and $\mathbf{v}_{\gamma}$, at $\mathcal{A}_{\beta e}$ and $\mathcal{A}_{\gamma e}$ can be ignored, and this is quite different than ignoring the boundary conditions for the spatial deviation velocities, $\tilde{\mathbf{v}}_{\beta}$ and $\tilde{\mathbf{v}}_{\gamma}$, at the entrances and exits of the macroscopic system. For many transport processes this approach is not useful; however, in this case the no-slip condition for the velocity requires that the velocity deviations be the same order of magnitude as the point velocities and both are dominated by the small length scale. This means that we can impose spatial periodicity directly on the point velocity, whereas periodicity can only be justified for the spatial deviation pressures and certainly not the point pressures. Because of this, we can introduce Equations (3.11) into Equations (3.9) to obtain the following two closure problems.

\section{Problem I}

$$
\begin{aligned}
& \nabla \cdot \mathbf{M}_{\beta \beta}=0, \quad \text { in the } \beta \text {-phase, } \\
& -\nabla \mathbf{m}_{\beta \beta}+\nabla^{2} \mathbf{M}_{\beta \beta}=\mathbf{I}, \quad \text { in the } \beta \text {-phase, }
\end{aligned}
$$


B.C.1 $\mathbf{M}_{\beta \beta}=0$, at $A_{\beta \sigma}$,

B.C.2 $\mathbf{M}_{\beta \beta}=\mathbf{M}_{\gamma \beta}$, at $A_{\beta \gamma}$,

B.C.3 $\mu_{\beta} \mathbf{n}_{\beta \cdot \gamma} \cdot\left[-\mathbf{I m}_{\beta \beta}+\left(\nabla \mathbf{M}_{\beta \beta}+\nabla \mathbf{M}_{\beta \beta}^{T}\right)\right]=$

$$
=\mu_{\gamma} \mathbf{n}_{\beta \gamma} \cdot\left[-\mathbf{I m}_{\gamma \beta}+\left(\nabla \mathbf{M}_{\gamma \beta}+\nabla \mathbf{M}_{\gamma \beta}^{T}\right)\right], \quad \text { at } A_{\beta \gamma},
$$

B.C.4 $\mathbf{M}_{\gamma \beta}=0$, at $A_{\gamma \sigma}$,

$$
\begin{aligned}
& -\nabla \mathbf{m}_{\gamma \beta}+\nabla^{2} \mathbf{M}_{\gamma \beta}=0, \quad \text { in the } \gamma \text {-phase, } \\
& \nabla \cdot \mathbf{M}_{\gamma \beta}=0, \quad \text { in the } \gamma \text {-phase, }
\end{aligned}
$$

Periodicity: $\quad \mathbf{m}_{\beta \beta}\left(\mathbf{r}+\ell_{i}\right)=\mathbf{m}_{\beta \beta}(\mathbf{r}), \quad \mathbf{M}_{\beta \beta}\left(\mathbf{r}+\ell_{i}\right)=\mathbf{M}_{\beta \beta}(\mathbf{r})$,

$$
\begin{aligned}
& i=1,2,3, \\
& \mathbf{m}_{\gamma \beta}\left(\mathbf{r}+\ell_{i}\right)=\mathbf{m}_{\gamma \beta}(\mathbf{r}), \quad \mathbf{M}_{\gamma \beta}\left(\mathbf{r}+\ell_{i}\right)=\mathbf{M}_{\gamma \beta}(\mathbf{r}), \\
& i=1,2,3 .
\end{aligned}
$$

Problem II

$$
\begin{aligned}
& \nabla \cdot \mathbf{M}_{\beta \gamma}=0, \quad \text { in the } \beta \text {-phase, } \\
& -\nabla \mathbf{m}_{\beta \gamma}+\nabla^{2} \mathbf{M}_{\beta \gamma}=0, \quad \text { in the } \beta \text {-phase, }
\end{aligned}
$$

B.C.2 $\quad \mathbf{M}_{\beta \gamma}=\mathbf{M}_{\gamma \gamma}$, at $A_{\beta \gamma}$,

B.C.4 $\mathbf{M}_{\gamma \gamma}=0$, at $A_{\gamma \sigma}$,

$$
\begin{aligned}
& -\nabla \mathbf{m}_{\gamma \gamma}+\nabla^{2} \mathbf{M}_{\gamma \gamma}=\mathbf{I}, \quad \text { in the } \gamma \text {-phase, } \\
& \nabla \cdot \mathbf{M}_{\gamma \gamma}=0, \quad \text { in the } \gamma \text {-phase, }
\end{aligned}
$$

Periodicity: $\quad \mathbf{m}_{\beta \gamma}\left(\mathbf{r}+\ell_{i}\right)=\mathbf{m}_{\beta \gamma}(\mathbf{r}), \quad \mathbf{M}_{\beta \gamma}\left(\mathbf{r}+\ell_{i}\right)=\mathbf{M}_{\beta \gamma}(\mathbf{r})$,

$$
\begin{aligned}
& i=1,2,3, \\
& \mathbf{m}_{\gamma \gamma}\left(\mathbf{r}+\ell_{i}\right)=\mathbf{m}_{\gamma \gamma}(\mathbf{r}), \quad \mathbf{M}_{\gamma \gamma}\left(\mathbf{r}+\ell_{i}\right)=\mathbf{M}_{\gamma \gamma}(\mathbf{r}), \\
& i=1,2,3 .
\end{aligned}
$$


Here we see exactly the same two problems as those given previously by Equations (2.10) and (2.11), and this leads to the results

$$
\begin{aligned}
& \mathbf{M}_{\beta \beta}=\mathbf{D}_{\beta \beta}^{0}, \\
& \mathbf{M}_{\gamma \beta}=\mathbf{D}_{\gamma \beta}^{0}, \\
& \mathbf{M}_{\beta \gamma}=\mathbf{D}_{\beta \gamma}^{0}, \\
& \mathbf{M}_{\gamma \gamma}=\mathbf{D}_{\gamma \gamma}^{0} .
\end{aligned}
$$

If we form the superficial average of the representations given by Equations (3.11a) and $(3.11 \mathrm{c})$, we obtain

$$
\begin{aligned}
& \left\langle\mathbf{v}_{\beta}\right\rangle=\frac{\varepsilon_{\beta}\left\langle\mathbf{M}_{\beta \beta}\right\rangle^{\beta}}{\mu_{\beta}} \cdot \Omega_{\beta}+\frac{\varepsilon_{\beta}\left\langle\mathbf{M}_{\beta \gamma}\right\rangle^{\beta}}{\mu_{\gamma}} \cdot \Omega_{\gamma}, \\
& \left\langle\mathbf{v}_{\gamma}\right\rangle=\frac{\varepsilon_{\gamma}\left\langle\mathbf{M}_{\gamma \beta}\right\rangle^{\gamma}}{\mu_{\beta}} \cdot \Omega_{\beta}+\frac{\varepsilon_{\gamma}\left\langle\mathbf{M}_{\gamma \gamma}\right\rangle^{\gamma}}{\mu_{\gamma}} \cdot \Omega_{\gamma}
\end{aligned}
$$

and on the basis of Equations (3.14) and Equations (2.10k) and (2.11k) we can write

$$
\begin{aligned}
& \varepsilon_{\beta}\left\langle\mathbf{M}_{\beta \beta}\right\rangle^{\beta}=-\mathbf{K}_{\beta \beta}^{*}, \\
& \varepsilon_{\beta}\left\langle\mathbf{M}_{\beta \gamma}\right\rangle^{\beta}=-\mathbf{K}_{\beta \gamma}^{*}, \\
& \varepsilon_{\gamma}\left\langle\mathbf{M}_{\gamma \beta}\right\rangle^{\gamma}=-\mathbf{K}_{\gamma \beta}^{*}, \\
& \varepsilon_{\gamma}\left\langle\mathbf{M}_{\gamma \gamma}\right\rangle^{\gamma}=-\mathbf{K}_{\gamma \gamma}^{*} .
\end{aligned}
$$

This means that Equations (3.15) are identical to Equations (1.4) and (1.5), and we have seen in this development a very short route between the physical problem given by Equations (1.6), the closure problems given by Equations (3.12) and Equations (3.13), and the macroscopic forms given by Equations (3.15). This represents a drastic reduction in analysis when compared to the previous efforts (Whitaker, 1986, 1994); however, one must remember that in this case we knew exactly what to look for and exactly how to find it.

\section{Symmetry and Reciprocity Relations}

In this section we show that the coupling permeability tensors satisfy a reciprocity relation given by

$$
\mu_{\beta} \mathbf{K}_{\beta \gamma}^{*}=\mu_{\gamma} \mathbf{K}_{\gamma \beta}^{* T}
$$


and using similar arguments one can prove that the dominant permeability tensors are symmetric

$$
\mathbf{K}_{\beta \beta}^{*}=\mathbf{K}_{\beta \beta}^{* T}, \quad \mathbf{K}_{\gamma \gamma}^{*}=\mathbf{K}_{\gamma \gamma}^{* T} .
$$

The latter is consistent with the proof that $\mathbf{K}_{\beta}$ and $\mathbf{K}_{\gamma}$ are symmetric (Whitaker, 1994), while the former represents a characteristic similar to that exhibited by the coupling thermal conductivity tensors that one encounters in two-equation models of heat conduction (Quintard and Whitaker, 1993). Equation (4.1) is consistent with the presentation of Auriault (1987).

The key to the proofs suggested by Equations (4.1) and (4.2) is to identify the vectors that are used to determine the four permeability tensors indicated by Equations (3.2k) and (3.3k). For example, if we wish to learn something about the properties of $\mathbf{K}_{\beta \gamma}^{*}$ we need to develop an expression that involves $\left\langle\mathbf{U}_{\beta}^{1}\right\rangle^{\beta}$. This leads us to form the scalar product of Equation (3.2b) with the vector $\mathbf{U}_{\beta}^{1}$ and then form the average to develop an equation for $\left\langle\mathbf{U}_{\beta}^{1}\right\rangle^{\beta}$. Because of the nature of the boundary condition given by Equations (3.2e), it is convenient to make use of the continuity equation given by Equation (3.2a) in order to express Equation (3.2b) as

$$
0=-\nabla P_{\beta}^{0}-\mathbf{e}_{0}+\nabla \cdot\left(\nabla \mathbf{U}_{\beta}^{0}+\nabla \mathbf{U}_{\beta}^{0 T}\right) .
$$

We now form the scalar product of this result with $\mathbf{U}_{\beta}^{1}$ to obtain

$$
\begin{aligned}
0= & -\nabla \cdot\left(P_{\beta}^{0} \mathbf{U}_{\beta}^{1}\right)-\mathbf{e}_{0} \cdot \mathbf{U}_{\beta}^{1}+\nabla \cdot\left[\left(\nabla \mathbf{U}_{\beta}^{0}+\nabla \mathbf{U}_{\beta}^{0 T}\right) \cdot \mathbf{U}_{\beta}^{1}\right]- \\
& -\left(\nabla \mathbf{U}_{\beta}^{0}+\nabla \mathbf{U}_{\beta}^{0 T}\right):\left(\nabla \mathbf{U}_{\beta}^{1}\right)^{T} .
\end{aligned}
$$

The superficial average of this result can be arranged as

$$
\begin{aligned}
& \frac{1}{\mathcal{V}} \int_{A_{\beta \gamma}} \mathbf{n}_{\beta \gamma} \cdot\left[-P_{\beta}^{0} \mathbf{U}_{\beta}^{1}+\left(\nabla \mathbf{U}_{\beta}^{0}+\nabla \mathbf{U}_{\beta}^{0 T}\right) \cdot \mathbf{U}_{\beta}^{1}\right] \mathrm{d} A- \\
& \quad-\frac{1}{\mathcal{V}} \int_{V_{\beta}}\left(\nabla \mathbf{U}_{\beta}^{0}+\nabla \mathbf{U}_{\beta}^{0 T}\right):\left(\nabla \mathbf{U}_{\beta}^{1}\right)^{T} \mathrm{~d} V=\mathbf{e}_{0} \cdot\left(\varepsilon_{\beta}\left\langle\mathbf{U}_{\beta}^{1}\right\rangle^{\beta}\right) .
\end{aligned}
$$

Here we have made use of the boundary condition given by Equation (3.2c) along with the periodicity condition that allows us to eliminate the area integrals over the entrances and exits of the $\beta$-phase contained within the averaging volume. On the basis of the definition of $\mathbf{K}_{\beta \gamma}^{*}$ given by Equation (3.3k), we can express Equation (4.5) as

$$
\begin{aligned}
& \frac{1}{\mathcal{V}} \int_{A_{\beta \gamma}} \mathbf{n}_{\beta \gamma} \cdot\left[-P_{\beta}^{0} \mathbf{U}_{\beta}^{1}+\left(\nabla \mathbf{U}_{\beta}^{0}+\nabla \mathbf{U}_{\beta}^{0 T}\right) \cdot \mathbf{U}_{\beta}^{1}\right] \mathrm{d} A- \\
& \quad-\frac{1}{\mathcal{V}} \int_{V_{\beta}}\left(\nabla \mathbf{U}_{\beta}^{0}+\nabla \mathbf{U}_{\beta}^{0 T}\right):\left(\nabla \mathbf{U}_{\beta}^{1}\right)^{T} \mathrm{~d} V=-\mathbf{e}_{0} \cdot \mathbf{K}_{\beta \gamma}^{*} \cdot \mathbf{e}_{1} .
\end{aligned}
$$


We now repeat this procedure by forming the scalar product of $\mathbf{U}_{\gamma}^{0}$ with Equation $(3.3 \mathrm{~g})$, integrating the result, and applying the boundary conditions to obtain

$$
\begin{aligned}
& \frac{1}{\mathcal{V}} \int_{A_{\gamma \beta}} \mathbf{n}_{\gamma \beta} \cdot\left[-P_{\gamma}^{1} \mathbf{U}_{\gamma}^{0}+\left(\nabla \mathbf{U}_{\gamma}^{1}+\nabla \mathbf{U}_{\gamma}^{1 T}\right) \cdot \mathbf{U}_{\gamma}^{0}\right] \mathrm{d} A- \\
& \quad-\frac{1}{\mathcal{V}} \int_{V_{\gamma}}\left(\nabla \mathbf{U}_{\gamma}^{1}+\nabla \mathbf{U}_{\gamma}^{1 T}\right):\left(\nabla \mathbf{U}_{\gamma}^{0}\right)^{T} \mathbf{d} V=-\mathbf{e}_{1} \cdot \mathbf{K}_{\gamma \beta}^{*} \cdot \mathbf{e}_{0} .
\end{aligned}
$$

In addition to forming the volume average of the scalar products associated with Equations (3.2b) and (3.3g), we will also need to perform the same type of operation with Equations (3.2g) and (3.3b). This leads us to a second equation for the $\beta$-phase

$$
\begin{gathered}
\frac{1}{\mathcal{V}} \int_{A_{\beta \gamma}} \mathbf{n}_{\beta \gamma} \cdot\left[-P_{\beta}^{1} \mathbf{U}_{\beta}^{0}+\left(\nabla \mathbf{U}_{\beta}^{1}+\nabla \mathbf{U}_{\beta}^{1 T}\right) \cdot \mathbf{U}_{\beta}^{0}\right] \mathrm{d} A- \\
-\frac{1}{\mathcal{V}} \int_{V_{\beta}}\left(\nabla \mathbf{U}_{\beta}^{1}+\nabla \mathbf{U}_{\beta}^{1 T}\right):\left(\nabla \mathbf{U}_{\beta}^{0}\right)^{T} \mathrm{~d} V=0
\end{gathered}
$$

and a second equation for the $\gamma$-phase

$$
\begin{gathered}
\frac{1}{\mathcal{V}} \int_{A_{\gamma \beta}} \mathbf{n}_{\gamma \beta} \cdot\left[-P_{\gamma}^{0} \mathbf{U}_{\gamma}^{1}+\left(\nabla \mathbf{U}_{\gamma}^{0}+\nabla \mathbf{U}_{\gamma}^{0 T}\right) \cdot \mathbf{U}_{\gamma}^{1}\right] \mathrm{d} A- \\
-\frac{1}{\mathcal{V}} \int_{V_{\gamma}}\left(\nabla \mathbf{U}_{\gamma}^{0}+\nabla \mathbf{U}_{\gamma}^{0 T}\right):\left(\nabla \mathbf{U}_{\gamma}^{1}\right)^{T} \mathrm{~d} V=0 .
\end{gathered}
$$

In order to connect these integrated forms, we need to make use of the boundary conditions given by Equations (3.2d) and (3.2e) along with the analogous boundary conditions in Problem I $\left(\mathrm{c}^{\prime}\right)$. Taking into account that $\mathbf{U}_{\beta}^{1}=\mathbf{U}_{\gamma}^{1}$ at the $\beta$ - $\gamma$ interface, we can form the scalar product of Equation (3.2e) with the velocity and integrate over the surface area $A_{\beta \gamma}$ to obtain

$$
\begin{aligned}
\mu_{\beta} & \left\{\frac{1}{\mathcal{V}} \int_{A_{\beta \gamma}} \mathbf{n}_{\beta \gamma} \cdot\left[-P_{\beta}^{0} \mathbf{U}_{\beta}^{1}+\left(\nabla \mathbf{U}_{\beta}^{0}+\nabla \mathbf{U}_{\beta}^{0 T}\right) \cdot \mathbf{U}_{\beta}^{1}\right] \mathrm{d} A\right\} \\
& =\mu_{\gamma}\left\{\frac{1}{\mathcal{V}} \int_{A_{\beta \gamma}} \mathbf{n}_{\beta \gamma} \cdot\left[-P_{\gamma}^{0} \mathbf{U}_{\gamma}^{1}+\left(\nabla \mathbf{U}_{\gamma}^{0}+\nabla \mathbf{U}_{\gamma}^{0 T}\right) \cdot \mathbf{U}_{\gamma}^{1}\right] \mathrm{d} A\right\} .
\end{aligned}
$$

Following the same procedure with Equation (3.3e) leads to

$$
\begin{aligned}
\mu_{\beta} & \left\{\frac{1}{\mathcal{V}} \int_{A_{\beta \gamma}} \mathbf{n}_{\beta \gamma} \cdot\left[-P_{\beta}^{1} \mathbf{U}_{\beta}^{0}+\left(\nabla \mathbf{U}_{\beta}^{1}+\nabla \mathbf{U}_{\beta}^{1 T}\right) \cdot \mathbf{U}_{\beta}^{0}\right] \mathrm{d} A\right\} \\
& =\mu_{\gamma}\left\{\frac{1}{\mathcal{V}} \int_{A_{\beta \gamma}} \mathbf{n}_{\beta \gamma} \cdot\left[-P_{\gamma}^{1} \mathbf{U}_{\gamma}^{0}+\left(\nabla \mathbf{U}_{\gamma}^{1}+\nabla \mathbf{U}_{\gamma}^{1 T}\right) \cdot \mathbf{U}_{\gamma}^{0}\right] \mathrm{d} A\right\} .
\end{aligned}
$$


We can use the first of these with Equations (4.6) and (4.9) to obtain

$$
\begin{aligned}
\mu_{\gamma} & \left\{\frac{1}{\mathcal{V}} \int_{V_{\gamma}}\left(\nabla \mathbf{U}_{\gamma}^{0}+\nabla \mathbf{U}_{\gamma}^{0 T}\right):\left(\nabla \mathbf{U}_{\gamma}^{1}\right)^{T} \mathrm{~d} V\right\}+ \\
& +\mu_{\beta}\left\{\frac{1}{\mathcal{V}} \int_{V_{\beta}}\left(\nabla \mathbf{U}_{\beta}^{0}+\nabla \mathbf{U}_{\beta}^{0 T}\right):\left(\nabla \mathbf{U}_{\beta}^{1}\right)^{T} \mathrm{~d} V\right\} \\
= & \mu_{\beta}\left(\mathbf{e}_{0} \cdot \mathbf{K}_{\beta \gamma}^{*} \cdot \mathbf{e}_{1}\right),
\end{aligned}
$$

while the use of Equation (4.11) with Equations (4.7) and (4.8) provides

$$
\begin{aligned}
\mu_{\beta}\{ & \left.\frac{1}{\mathcal{V}} \int_{V_{\beta}}\left(\nabla \mathbf{U}_{\beta}^{1}+\nabla \mathbf{U}_{\beta}^{1 T}\right):\left(\nabla \mathbf{U}_{\beta}^{0}\right)^{T} \mathrm{~d} V\right\}+ \\
& +\mu_{\gamma}\left\{\frac{1}{\mathcal{V}} \int_{V_{\gamma}}\left(\nabla \mathbf{U}_{\gamma}^{1}+\nabla \mathbf{U}_{\gamma}^{1 T}\right):\left(\nabla \mathbf{U}_{\gamma}^{0}\right)^{T} \mathrm{~d} V\right\} \\
= & \mu_{\gamma}\left(\mathbf{e}_{1} \cdot \mathbf{K}_{\gamma \beta}^{*} \cdot \mathbf{e}_{0}\right) .
\end{aligned}
$$

At this point we need to make use of the orthogonality characteristics associated with the irreducible parts of second order tensors to conclude that

$$
\left(\mathbf{A}+\mathbf{A}^{T}\right):\left(\mathbf{B}^{T}\right)=\frac{1}{2}\left(\mathbf{A}+\mathbf{A}^{T}\right):\left(\mathbf{B}+\mathbf{B}^{T}\right) .
$$

From this relation we find that Equations (4.12) and (4.13) provide

$$
\mu_{\beta}\left(\mathbf{e}_{0} \cdot \mathbf{K}_{\beta \gamma}^{*} \cdot \mathbf{e}_{1}\right)=\mu_{\gamma}\left(\mathbf{e}_{1} \cdot \mathbf{K}_{\gamma \beta}^{*} \cdot \mathbf{e}_{0}\right) .
$$

Since the unit vectors $\mathbf{e}_{0}$ and $\mathbf{e}_{1}$ are arbitrary, this result leads us to

$$
\mu_{\beta} \mathbf{K}_{\beta \gamma}^{*}=\mu_{\gamma} \mathbf{K}_{\gamma \beta}^{* T}
$$

and this constraint on the coupling permeability tensors was listed earlier as Equation (4.1).

In order to prove the symmetry conditions indicated by Equations (4.2), it is best to return to Problems $\mathrm{I}(\mathrm{b})$ and $\mathrm{I}\left(\mathrm{b}^{\prime}\right)$ and form the scalar products with two arbitrary unit vectors. One then follows the type of procedure given by Equations (4.3) through (4.15) to arrive at the symmetry conditions for the dominant permeability tensors. The details have been presented by Whitaker (1994, Sec. 5).

Given the reciprocity relation indicated by Equation (4.16), one can return to the matter of the viscous drag tensors in Equations (1.1) and (1.2) and explore the relation between $\mathbf{K}_{\beta \gamma}$ and $\mathbf{K}_{\gamma \beta}$. After some algebraic effort one finds that the two viscous drag tensors are related by

$$
\mu_{\beta} \mathbf{K}_{\beta \gamma} \cdot \mathbf{K}_{\gamma}=\mu_{\gamma} \mathbf{K}_{\beta} \cdot \mathbf{K}_{\gamma \beta}^{T}
$$


and this means that there are only three independent tensors to be determined theoretically or experimentally. Similar conclusions have been obtained by Auriault (1987) on the basis of local problems defining the tensors that are similar to the problems given by Equations (3.12) and (3.13). Arguments provided by Kalaydjian (1988) are taken from thermodynamics of irreversible processes as applied to porous systems; however, the use of Casimir-Onsager-like relations has not been proven at the microscopic level for the problem under consideration. More recently, it has been indicated (Bentsen, 1994) that the coupling terms are not necessarily equal. This result is based on indirect measurements and, as we pointed out earlier, indirect measurements may be affected by the existence of two different interface configurations at the same saturation. Conclusions extracted from two different experiments are somewhat questionable, and at this point in time they are not supported by direct measurements. On the other hand, our results are derived for the same interface geometry.

\section{Conclusions}

In this paper we have illustrated the relations between the viscous drag tensors and the coupling permeability tensors for two-phase flow in homogeneous porous media, and we have shown how both sets of tensors can be extracted from the same two closure problems. Symmetry and reciprocity relations among the permeability and drag tensors have been discussed and a method of solving the closure problem has been presented.

\section{Appendix. Reciprocity Relation}

In Section 4 we proved the reciprocity relation given by

$$
\mu_{\beta} \mathbf{K}_{\beta \gamma}^{*}=\mu_{\beta} \mathbf{K}_{\gamma \beta}^{* T} \quad \text { or } \mu_{\gamma} \mathbf{K}_{\gamma \beta}^{*}=\mu_{\beta} \mathbf{K}_{\beta \gamma}^{* T}
$$

and in this appendix we wish to show how this result can be used to establish a relation between the two viscous drag tensors, $\mathbf{K}_{\beta \gamma}$ and $\mathbf{K}_{\gamma \beta}$, and we wish to explore the problem of estimating tensors on the basis of the closure problems. From Equations (1.1), (1.2), (1.4), and (1.5) we can quickly deduce the following relations

$$
\begin{aligned}
& \mathbf{K}_{\beta \beta}^{*}=\left(\mathbf{I}-\mathbf{K}_{\beta \gamma} \mathbf{K}_{\gamma \beta}\right)^{-1} \cdot \mathbf{K}_{\beta}, \\
& \mathbf{K}_{\beta \gamma}^{*}=\left(\mathbf{I}-\mathbf{K}_{\gamma \beta} \mathbf{K}_{\beta \gamma}\right)^{-1} \cdot \mathbf{K}_{\beta \gamma} \cdot \mathbf{K}_{\gamma}, \\
& \mathbf{K}_{\gamma \gamma}^{*}=\left(\mathbf{I}-\mathbf{K}_{\gamma \beta} \mathbf{K}_{\beta \gamma}\right)^{-1} \cdot \mathbf{K}_{\gamma}, \\
& \mathbf{K}_{\gamma \beta}^{*}=\left(\mathbf{I}-\mathbf{K}_{\gamma \beta} \mathbf{K}_{\beta \gamma}\right)^{-1} \cdot \mathbf{K}_{\gamma \beta} \cdot \mathbf{K}_{\beta},
\end{aligned}
$$

From this work, and from the earlier study of Whitaker (1994), we have the following symmetry conditions

$$
\mathbf{K}_{\beta \beta}^{*}=\mathbf{K}_{\beta \beta}^{* T},
$$




$$
\begin{aligned}
& \mathbf{K}_{\beta}=\mathbf{K}_{\beta}^{T}, \\
& \mathbf{K}_{\gamma \gamma}^{*}=\mathbf{K}_{\gamma \gamma}^{* T}, \\
& \mathbf{K}_{\gamma}=\mathbf{K}_{\gamma}^{T} .
\end{aligned}
$$

In order to use Equations (A.2) through (A.9) along with Equation (A.1) to develop a relation between $\mathbf{K}_{\beta \gamma}$ and $\mathbf{K}_{\gamma \beta}$, we need the following results for second order tensors

$$
\begin{aligned}
& (\mathbf{A} \cdot \mathbf{B})^{T}=\mathbf{B}^{T} \cdot \mathbf{A}^{T}, \\
& \left(\mathbf{A}^{-1}\right)^{T}=\left(\mathbf{A}^{T}\right)^{-1}, \\
& (\mathbf{A}+\mathbf{B})^{T}=\mathbf{A}^{T}+\mathbf{B}^{T}, \\
& (\mathbf{A} \cdot \mathbf{B})^{-1}=\mathbf{B}^{-1} \cdot \mathbf{A}^{-1} .
\end{aligned}
$$

On the basis of the symmetry condition given by Equation (A.6), we see that Equation (A.2) provides

$$
\left(\mathbf{I}-\mathbf{K}_{\beta \gamma} \cdot \mathbf{K}_{\gamma \beta}\right)^{-1} \cdot \mathbf{K}_{\beta}=\left[\left(\mathbf{I}-\mathbf{K}_{\beta \gamma} \cdot \mathbf{K}_{\gamma \beta}\right)^{-1} \cdot \mathbf{K}_{\beta}\right]^{T}
$$

and use of Equations (A.10) through (A.12) with the right-hand side of this result leads to

$$
\left(\mathbf{I}-\mathbf{K}_{\beta \gamma} \cdot \mathbf{K}_{\gamma \beta}\right)^{-1} \cdot \mathbf{K}_{\beta}=\mathbf{K}_{\beta} \cdot\left(\mathbf{I}-\mathbf{K}_{\gamma \beta}^{T} \cdot \mathbf{K}_{\beta \gamma}^{T}\right)^{-1} .
$$

If we take the inverse of this result and apply Equation (A.13), we find

$$
\mathbf{K}_{\beta}^{-1} \cdot\left(\mathbf{I}-\mathbf{K}_{\beta \gamma} \cdot \mathbf{K}_{\gamma \beta}\right)=\left(\mathbf{I}-\mathbf{K}_{\gamma \beta}^{T} \cdot \mathbf{K}_{\beta \gamma}^{T}\right) \cdot \mathbf{K}_{\beta}^{-1},
$$

which can be simplified to

$$
\mathbf{K}_{\beta}^{-1} \cdot \mathbf{K}_{\beta \gamma} \cdot \mathbf{K}_{\gamma \beta}=\mathbf{K}_{\gamma \beta}^{T} \cdot \mathbf{K}_{\beta \gamma}^{T} \cdot \mathbf{K}_{\beta}^{-1} .
$$

Subsequently we will find it convenient to multiply this result by $\mathbf{K}_{\beta}$ so that we can make use of the following form

$$
\mathbf{K}_{\beta \gamma} \cdot \mathbf{K}_{\gamma \beta} \cdot \mathbf{K}_{\beta}=\mathbf{K}_{\beta} \cdot \mathbf{K}_{\gamma \beta}^{T} \cdot \mathbf{K}_{\beta \gamma}^{T} .
$$

We can repeat this development starting with Equations (A.4) and (A.8) in order to obtain a result that is analogous to Equation (A.18). This is given by

$$
\mathbf{K}_{\gamma \beta} \cdot \mathbf{K}_{\beta \gamma} \cdot \mathbf{K}_{\gamma}=\mathbf{K}_{\gamma} \cdot \mathbf{K}_{\beta \gamma}^{T} \cdot \mathbf{K}_{\gamma \beta}^{T}
$$


and we are now ready to introduce the reciprocity relation that was derived in Section 4.

If we substitute Equations (A.3) and (A.5) into the second of Equations (A.1) and make use of Equations (A.10) through (A.12), we obtain

$$
\begin{aligned}
& \mu_{\gamma}\left(\mathbf{I}-\mathbf{K}_{\gamma \beta} \cdot \mathbf{K}_{\beta \gamma}\right)^{-1} \cdot \mathbf{K}_{\gamma \beta} \cdot \mathbf{K}_{\beta \gamma} \\
& \quad=\mu_{\beta} \mathbf{K}_{\gamma} \cdot \mathbf{K}_{\beta \gamma}^{T} \cdot\left(\mathbf{I}-\mathbf{K}_{\gamma \beta}^{T} \cdot \mathbf{K}_{\beta \gamma}^{T}\right)^{-1} .
\end{aligned}
$$

Use of Equation (A.13) allows us to express the inverse of this result as

$$
\begin{aligned}
& \mu_{\beta} \mathbf{K}_{\beta}^{-1} \cdot \mathbf{K}_{\gamma \beta}^{-1} \cdot\left(\mathbf{I}-\mathbf{K}_{\gamma \beta} \cdot \mathbf{K}_{\beta \gamma}\right) \\
& \quad=\mu_{\gamma}\left(\mathbf{I}-\mathbf{K}_{\gamma \beta}^{T} \cdot \mathbf{K}_{\beta \gamma}^{T}\right) \cdot\left(\mathbf{K}_{\beta \gamma}^{T}\right)^{-1} \cdot \mathbf{K}_{\gamma}^{-1},
\end{aligned}
$$

or in the equivalent form given by

$$
\mu_{\beta} \mathbf{K}_{\beta}^{-1} \cdot\left(\mathbf{K}_{\gamma \beta}^{-1}-\mathbf{K}_{\beta \gamma}\right)=\mu_{\gamma}\left[\left(\mathbf{K}_{\beta \gamma}^{T}\right)^{-1}-\mathbf{K}_{\gamma \beta}^{T}\right] \cdot \mathbf{K}_{\gamma}^{-1} .
$$

This result can be pre-multiplied by $\mathbf{K}_{\beta}$ and post-multiplied by $\mathbf{K}_{\gamma}$ to obtain

$$
\mu_{\beta}\left(\mathbf{K}_{\gamma \beta}^{-1}-\mathbf{K}_{\beta \gamma}\right) \cdot \mathbf{K}_{\gamma}=\mu_{\gamma} \mathbf{K}_{\beta} \cdot\left[\left(\mathbf{K}_{\beta \gamma}^{T}\right)^{-1}-\mathbf{K}_{\gamma \beta}^{T}\right] .
$$

In order to eliminate the inverse of the transpose on the right-hand side of this result, we multiply by $\mathbf{K}_{\beta \gamma}^{T}$ leading to

$$
\mu_{\beta}\left(\mathbf{K}_{\gamma \beta}^{-1}-\mathbf{K}_{\beta \gamma}\right) \cdot \mathbf{K}_{\gamma} \cdot \mathbf{K}_{\beta \gamma}^{T}=\mu_{\gamma}\left(\mathbf{K}_{\beta}-\mathbf{K}_{\beta} \cdot \mathbf{K}_{\gamma \beta}^{T} \cdot \mathbf{K}_{\beta \gamma}^{T}\right) .
$$

We now see that the second term on the right-hand side has appeared previously in Equation (A.18) and we can use that result to express Equation (A.24) as

$$
\mu_{\beta}\left(\mathbf{K}_{\gamma \beta}^{-1}-\mathbf{K}_{\beta \gamma}\right) \cdot \mathbf{K}_{\gamma} \cdot \mathbf{K}_{\beta \gamma}^{T}=\mu_{\gamma}\left(\mathbf{I}-\mathbf{K}_{\beta \gamma} \cdot \mathbf{K}_{\gamma \beta}\right) \cdot \mathbf{K}_{\beta} .
$$

Keeping Equation (A.19) in mind, we multiply by $\mathbf{K}_{\gamma \beta}^{T}$ to provide

$$
\begin{array}{r}
\mu_{\beta}\left(\mathbf{K}_{\gamma \beta}^{-1}-\mathbf{K}_{\beta \gamma}\right) \cdot \mathbf{K}_{\gamma} \cdot \mathbf{K}_{\beta \gamma}^{T} \cdot \mathbf{K}_{\gamma \beta}^{T} \\
=\mu_{\gamma}\left(\mathbf{I}-\mathbf{K}_{\beta \gamma} \cdot \mathbf{K}_{\gamma \beta}\right) \cdot \mathbf{K}_{\beta} \cdot \mathbf{K}_{\gamma \beta}^{T}
\end{array}
$$

and when Equation (A.19) is substituted into this result we obtain

$$
\begin{gathered}
\mu_{\beta}\left(\mathbf{K}_{\gamma \beta}^{-1}-\mathbf{K}_{\beta \gamma}\right) \cdot \mathbf{K}_{\gamma \beta} \cdot \mathbf{K}_{\beta \gamma} \cdot \mathbf{K}_{\gamma} \\
=\mu_{\gamma}\left(\mathbf{I}-\mathbf{K}_{\beta \gamma} \cdot \mathbf{K}_{\gamma \beta}\right) \cdot \mathbf{K}_{\beta} \cdot \mathbf{K}_{\gamma \beta}^{T} .
\end{gathered}
$$

Carrying out the obvious multiplication on the left-hand side yields

$$
\begin{aligned}
& \mu_{\beta}\left(\mathbf{I}-\mathbf{K}_{\beta \gamma} \cdot \mathbf{K}_{\gamma \beta}\right) \cdot \mathbf{K}_{\beta \gamma} \cdot \mathbf{K}_{\gamma} \\
& \quad=\mu_{\gamma}\left(\mathbf{I}-\mathbf{K}_{\beta \gamma} \cdot \mathbf{K}_{\gamma \beta}\right) \cdot \mathbf{K}_{\beta} \cdot \mathbf{K}_{\gamma \beta}^{T}
\end{aligned}
$$


and we need only multiply this result by $\left(\mathbf{I}-\mathbf{K}_{\beta \gamma} \cdot \mathbf{K}_{\gamma \beta}\right)^{-1}$ to obtain

$$
\mu_{\beta} \mathbf{K}_{\beta \gamma} \cdot \mathbf{K}_{\gamma}=\mu_{\gamma} \mathbf{K}_{\beta} \cdot \mathbf{K}_{\gamma \beta}^{T} \text {. }
$$

This result indicates that the viscous drag tensors that appear in Equations (1.1) and (1.2) are not independent, and it also suggests that the relation between these two tensors is something quite different than the order of magnitude analysis (Whitaker, 1994) that led to

$$
\mathbf{K}_{\beta \gamma} \cdot \mathbf{K}_{\gamma \beta}=\mathbf{O}(\mathbf{I})
$$

In the absence of detailed calculations, one cannot be certain that Equations (A.29) and (A.30) are contradictory; however, we are now in a position to test the order of magnitude analysis in terms of the rigorous result given by Equation (A.1).

\section{ORDER OF MAGNITUDE ANALYSIS}

In order to illustrate the difficulties associated with estimating coefficients on the basis of closure problems, we consider the reciprocity relation

$$
\mu_{\beta} \mathbf{K}_{\beta \gamma}^{*}=\mu_{\gamma} \mathbf{K}_{\gamma \beta}^{* T}
$$

and the two closure problems that are used to determine the coupling permeability tensors. The two closure problems are given by Equations (3.12) and (3.13), and the coupling permeability tensors are defined in terms of the solutions to these problems as follows

$$
\mathbf{K}_{\beta \gamma}^{*}=-\varepsilon_{\beta}\left\langle\mathbf{M}_{\beta \gamma}\right\rangle^{\beta}, \quad \mathbf{K}_{\gamma \beta}^{*}=-\varepsilon_{\gamma}\left\langle\mathbf{M}_{\gamma \beta}\right\rangle^{\beta} .
$$

In order to estimate the tensor $\mathbf{M}_{\beta \gamma}$, we return to Equations (3.13) and note that the single, non-homogeneous term in that boundary-value problem is the unit tensor in Equation (3.13g). If, in that equation, we assume that

$$
\nabla^{2} \mathbf{M}_{\gamma \gamma} \geqslant \mathbf{O}\left(\nabla \mathbf{m}_{\gamma \gamma}\right)
$$

we immediately estimate $\mathbf{M}_{\gamma \gamma}$ as

$$
\mathbf{M}_{\gamma \gamma}=\mathbf{O}\left(\ell_{\gamma}^{2}\right)
$$

In order to use this to estimate $\mathbf{M}_{\beta \gamma}$, we could employ the boundary condition given by Equation (3.13d) to conclude that

$$
\mathbf{M}_{\beta \gamma}=\mathbf{O}\left(\mathbf{M}_{\gamma \gamma}\right)=\mathbf{O}\left(\ell_{\gamma}^{2}\right) .
$$


This estimate completely ignores the stress boundary condition given by Equation (3.13e), and on the basis of Equation (A.33) one might be willing to interpret Equation (3.13e) subject to the following estimates

$$
\nabla \mathbf{M}_{\gamma \gamma} \geqslant \mathbf{O}\left(\mathbf{m}_{\gamma \gamma}\right), \quad \nabla \mathbf{M}_{\beta \gamma} \geqslant \mathbf{O}\left(\mathbf{m}_{\beta \gamma}\right)
$$

This would lead us to conclude that

$$
\mu_{\beta} \nabla \mathbf{M}_{\beta \gamma}=\mathbf{O}\left(\mu_{\gamma} \nabla \mathbf{M}_{\gamma \gamma}\right)
$$

and we estimate the gradients in the usual manner to obtain

$$
\left(\mu_{\beta} \frac{\Delta \mathbf{M}_{\beta \gamma}}{\ell_{\beta}}\right)=\mathbf{O}\left(\mu_{\gamma} \frac{\Delta \mathbf{M}_{\gamma \gamma}}{\ell_{\gamma}}\right) .
$$

Since both $\mathbf{M}_{\beta \gamma}$ and $\mathbf{M}_{\gamma \gamma}$ are zero at the fluid-solid interfaces, the characteristic change in these tensors should be on the order of the tensors themselves, thus we write

$$
\Delta \mathbf{M}_{\beta \gamma}=\mathbf{O}\left(\mathbf{M}_{\beta \gamma}\right), \quad \text { and } \Delta \mathbf{M}_{\gamma \gamma}=\mathbf{O}\left(\mathbf{M}_{\gamma \gamma}\right) .
$$

Use of Equations (A.35) and (A.39) in Equation (A.38) leads to the following estimate for $\mathbf{M}_{\beta \gamma}$

$$
\mu_{\beta} \mathbf{M}_{\beta \gamma}=\mathbf{O}\left(\mu_{\gamma} \ell_{\gamma} \ell_{\beta}\right)
$$

When Equations (A.35) and (A.40) are used in the first of Equations (A.32), we obtain two estimates indicated by

$$
\mu_{\beta} \mathbf{K}_{\beta \gamma}^{*}=\mathbf{O}\left\{\begin{array}{l}
\varepsilon_{\beta} \mu_{\beta} \ell_{\gamma}^{2} \\
\varepsilon_{\beta} \mu_{\gamma} \ell_{\beta} \ell_{\gamma}
\end{array} .\right.
$$

In order to estimate $\mathbf{K}_{\gamma \beta}^{*}$ as defined in Equation(A.32), we make use of the boundary value problem given by Equations (3.12) and repeat the above analysis to obtain

$$
\mu_{\gamma} \mathbf{K}_{\gamma \beta}^{*}=\mathbf{O}\left\{\begin{array}{l}
\varepsilon_{\gamma} \mu_{\gamma} \ell_{\beta}^{2} \\
\varepsilon_{\gamma} \mu_{\beta} \ell_{\gamma} \ell_{\beta}
\end{array} .\right.
$$

Here we can see that these two estimates are not at all consistent with the reciprocity relation given by Equation (A.31).

The reason for the failure to obtain good estimates for $\mathbf{K}_{\beta \gamma}^{*}$ and $\mathbf{K}_{\gamma \beta}^{*}$ is difficult to identify. The estimate of $\mathbf{M}_{\gamma \gamma}$ given by Equation (A.34) seems reasonable, especially in light of the fact that this tensor is zero at the $\gamma-\sigma$ interface. In order to connect $\mathbf{M}_{\gamma \gamma}$ with $\mathbf{M}_{\beta \gamma}$ we are forced to use one or both of the interfacial 
boundary conditions represented by Equations (3.13d) and (3.13e), and it must be our treatment of these boundary conditions that leads to the contradiction between Equations (A.41) and (A.42) and the exact result given by Equation (A.1). In an earlier study of the closure problem for two-phase flow, Whitaker (1994) used order of magnitude analysis similar to that presented here to conclude that $\mathbf{K}_{\beta \gamma} \cdot \mathbf{K}_{\gamma \beta}=$ $\mathbf{O}(1)$ and at this point in time we cannot consider this to be a reliable relation between the two viscous drag tensors.

\section{Acknowledgement}

This work was done while S.W. was a visitor at the Laboratoire Energétique et Phénomènes de Transfert. The support from L.E.P.T.-ENSAM is greatly appreciated.

\section{References}

Auriault, J.-L.: 1987, Nonsaturated deformable porous media: Quasisatics, Transport in Porous Media 2, 45-64.

Bacri, J. C., Chaouche, M., and Salin, D.: 1990, Modèle simple de perméabilités relatives croisées, C. R. Acad. Sc. Paris Sér. II 311, 591-597.

Baveye, P. and Sposito, G.: 1984, The operational significance of the continuum hypothesis in the theory of water movement through soils and aquifers, Water Resour. Res. 20, 521-530.

Bentsen, R. G.: 1994, An investigation into whether the nondiagonal mobility coefficients which arise in coupled, two phase flow are equal, Transport in Porous Media 14, 23-32.

Bentsen, R. G. and Manai, A. A.: 1993, On the use of conventional co-current and counter-current effective permeabilities to estimate the four generalized permeability coefficients which arise in coupled, two-phase flow, Transport in Porous Media 11, 243-262.

Bensoussan, A., Lions, J. L. and Papanicolaou, G.: 1978, Asymptotic Analysis for Periodic Structures, North-Holland, Amsterdam, The Netherlands.

Bourbiaux, B. J. and Kalaydjian, F. J.: 1990, Experimental study of co-current and counter-current flows in natural porous media, SPERE 5, 361-368.

Carbonell, R. G. and Whitaker, S.: 1984, Heat and mass transfer in porous media, in: J. Bear and M. Y. Corapcioglu (eds), Fundamentals of Transport Phenomena in Porous Media, Martinus Nijhoff, Dordrecht, The Netherlands, pp. 123-198.

Danis, M. and Jacquin, C.: 1983, Influence du contraste de viscosités sur les perméabilités relatives lors du drainage: Expérimentation et modélisation, Revue de l'Institut Français du Pétrole 38, 723-733.

Danis, M. and Quintard, M.: 1984, Modélisation d'un écoulement diphasique dans une succession de pores, Revue de l'Institut Français du Pétrole 39, 37-46.

de Gennes, P. G., 1983, Theory of slow biphasic flows in porous media, Physico Chem. Hydrody. 4, $175-185$.

Goode, P. A. and Ramakrishnan, T. S.: 1993, Momentum transfer across fluid-fluid interfaces in porous media: a network model, AIChE J. 39, 1124-1134.

Howes, F. A. and Whitaker, S.: 1985, The spatial averaging theorem revisited, Chem. Engng. Sci. 40, $1387-1392$.

Kalaydjian, F.: 1988, Couplage entre phases fluides dans les écoulements diphasiques en milieu poreux, Thèse de l'Université Bordeaux I.

Kalaydjian, F.: 1990, Origin and quantification of coupling between relative permeabilities for twophase flows in porous media, Transport in Porous Media 5, 215-229.

Kalaydjian, F. and Legait, B.: 1987a, Écoulement lent à contre-courant de deux fluides non miscibles dans un capillaire présentant un rétrécissement, C. R. Acad. Sc. Paris Sér: II 304, 869-872. 
Kalaydjian, F. and Legait, B.: 1987b, Perméabilités relatives couplées dans des écoulements en capillaire et en milieu poreux, C. R. Acad. Sc. Paris Sér. II 304, 1035-1038.

Kalaydjian, F. and Legait, B.: 1988, Effets de la géométrie des pores et de la mouillabilité sur le déplacement diphasique à contre-courant en capillaire et en milieu poreux, Rev. Phys. Appl. 23, 1071-1081.

Mannseth, T.: 1991, Commentary on 'origin and quantification of coupling between relative permeabilities for two-phase flows in porous media' by F. Kalaydjian, Transport in Porous Media 6, 469-471.

Marle, C. M.: 1982, On macroscopic equations governing multiphase flow with diffusion and chemical reactions in porous media, Int. J. Engng. Sci. 50, 643-662.

Quintard, M. and Whitaker, S.: 1987, Écoulement monophasique en milieu poreux: Effet des hétérogénéités locales, J. Méc. Théor. Appl. 6, 691-726.

Quintard, M. and Whitaker, S.: 1988, Two-phase flow in heterogeneous porous media: The method of large-scale averaging, Transport in Porous Media 3, 357-413.

Quintard, M. and Whitaker, S.: 1990, Two-phase flow in heterogeneous porous media I: The influence of large spatial and temporal gradients, Transport in Porous Media 5, 341-379.

Quintard, M. and Whitaker, S.: 1993, One and two-equation models for transient diffusion processes in two-phase systems, in: Advances in Heat Transfer, Vol. 23, Academic Press, New York, pp. $369-465$.

Quintard, M. and Whitaker, S.: 1994, Transportin ordered and disordered porous media I: The cellular average and the use of weighting functions, Transport in Porous Media 14, 163-177.

Quintard, M. and Whitaker, S.: 1994, Transport in ordered and disordered porous media II: Generalized volume averaging, Transport in Porous Media 14, 179-206.

Raats, P. A. C. and Klute, A.: 1968, Transport in soils: The balance of momentum, Soil Sci. Soc. Amer. Proc. 32, 161-166.

Rose, W.: 1988, Measuring transport coefficients necessary for the description of coupled two-phase flow of immiscible fluids in porous media, Transport in Porous Media 3, 163-171.

Rose, W.: 1989, Data interpretation problems to be expected in the study of coupled fluid flow in porous media, Transport in Porous Media 4, 185-189.

Rothman, D. H.: 1990, Macroscopic laws for immiscible two-phase flow in porous media: Results from numerical experiments, J. Geophys. Res. 95, 8663-8674.

Torres, F. E.: 1987, Closure of the governing equations for immiscible, two-phase flow: A research comment, Transport in Porous Media 2, 383-393.

Whitaker, S.: 1986, Flow in porous media II: The governing equations for immiscible two-phase flow, Transport in Porous Media 1, 105-125.

Whitaker, S.: 1994, The closure problem for two-phase flow in homogeneous porous media, Chem. Engng. Sci. 49, 765-780.

Zarcone, C.: 1994, Etude du couplage visqueux en milieu poreux: mesure des perméabilités relatives croisées, Thèse de l'Institut Polytechnique National de Toulouse.

Zarcone, C. and Lenormand, R.: 1994, Détermination expérimentale du couplage visqueux dans les écoulements diphasiques en milieu poreux, C. R. Acad. Sci. Paris Sér. II 318, 1429-1435. 